\title{
Self-Assembled Dual-Targeted Epirubicin-Hybrid Polydopamine Nanoparticles for Combined Chemo-Photothermal Therapy of Triple-Negative Breast Cancer
}

This article was published in the following Dove Press journal:

International Journal of Nanomedicine

\begin{abstract}
Xiang $\mathrm{Li}^{1, *}$
Qian Zou' ${ }^{1} *$

Jing Zhang ${ }^{2}$

Peng Zhang ${ }^{2}$

Xiong Zhou'

Satya Siva Kishan Yalamarty ${ }^{3}$

Xinli Liang $\mathbb{D}^{2}$

Yali Liu ${ }^{4}$

Qin Zheng ${ }^{2}$

Jianqing $\mathrm{Gao}^{5}$

'State Key Laboratory of Innovative Drug and Efficient Energy-Saving Pharmaceutical Equipment, Jiangxi University of Traditional Chinese Medicine, Nanchang 330006, Jiangxi, People's Republic of China; ${ }^{2}$ Key Laboratory of Modern Preparation of TCM, Ministry of Education, Jiangxi University of Traditional Chinese Medicine, Nanchang 330004, Jiangxi, People's Republic of China; ${ }^{3}$ Center for Pharmaceutical Biotechnology and Nanomedicine, Northeastern University, Boston, MA 02 I I5, USA; ${ }^{4}$ College of Science and Technology, Jiangxi University of Traditional Chinese Medicine, Nanchang 330004, Jiangxi, People's Republic of China; ${ }^{5}$ Institute of Pharmaceutics, College of Pharmaceutical Sciences, Zhejiang University, Hangzhou 310058, Zhejiang, People's Republic of China

*These authors contributed equally to this work
\end{abstract}

Correspondence: Jing Zhang

Key Laboratory of Modern Preparation of TCM, Ministry of Education, Jiangxi University of Traditional Chinese

Medicine, Nanchang 330004, Jiangxi,

People's Republic of China

$\mathrm{Tel} / \mathrm{Fax}+86$ 79I 87II8658

Email20101036@jxutcm.edu.cn
Purpose: Folic acid and cyclic arginylglycylaspartic acid peptides were introduced to the surface of negatively charged lipid-coated hybrid polydopamine-cysteine cores for the delivery of epirubicin (EPI) (E/PCF-NPs). The combined chemo-photothermal therapy using E/PCF-NPs for triple-negative breast cancer was evaluated.

Materials and Methods: The temperature elevation and thermal toxicity of nanoparticles were studied. The morphology and properties of E/PCF-NPs were characterized by transmission electron microscopy, scanning electron microscopy, and atomic force microscopy. Physicochemical properties, including particle size, zeta potential, drug loading, entrapment efficiency (EE\%), stability and in vitro release, were determined. The cell viability, reactive oxygen species (ROS) levels, ratios of oxidized nicotinamide adenine dinucleotide to its reduced form $\left(\mathrm{NAD}^{+} / \mathrm{NADH}\right)$, apoptosis assays, and cellular uptake of E/PCF-NPs were determined on 4T1 cells. Pharmacokinetic studies and tissue distributions were performed and detected by an ultra-high performance liquid chromatography/mass spectrometry system. The antitumor effects of E/PCF-NPs under near-infrared (NIR) laser irradiation were also evaluated.

Results: The sphere-like morphology of E/PCF-NPs showed a high EE\%, uniform size of 106.7 $\mathrm{nm}$, remarkable stability, and highly improved cytotoxicity under NIR laser, when compared to that of photothermal treatment alone. In vitro release of EPI from E/PCF-NPs was $\mathrm{pH}$ sensitive, and a greater response was achieved under NIR laser irradiation. Compared to chemotherapy or photothermal treatment alone, the combined treatment in vitro significantly inhibited the survival rate of $4 \mathrm{~T} 1$ cells to $17.7 \%$, induced $\mathrm{ROS}$ generation, and reduced $\mathrm{NAD}^{+} / \mathrm{NADH}$ significantly. Treatment with E/PCF-NPs under irradiation induced 4T1 cell apoptosis in approximately $93.6 \%$ cells. In vitro cellular uptake of E/PCF-NPs was time-dependent. The long-circulating and higher tumor accumulation of E/PCF-NPs resulted in complete ablation of breast tumor tissue through the enhanced photothermal effect by NIR laser irradiation-mediated cell apoptosis.

Conclusion: E/PCF-NPs show enhanced anti-cancer effects due to synergistic effects of chemotherapy with photothermal therapy and may be potential therapeutic agents for cancer treatment.

Keywords: polydopamine nanoparticles, L-cysteine, epirubicin, pharmacokinetics, triplenegative breast cancer

\section{Introduction}

Triple-negative breast cancer (TNBC) is characterized by a lack of immunohistochemical expression of the estrogen and progesterone receptors and human epidermal growth 
factor receptor 2, and accounts for $15-20 \%$ of all breast cancer (BC) cases. ${ }^{1}$ The TNBC subtype is associated with higher aggression, poorer prognosis, higher visceral and central nervous system metastases, and rates of recurrence despite treatment compared to other subtypes. ${ }^{2}$ The median survival of patients with advanced TNBC is, at best, 12 months, which is significantly shorter than the survival for other subtypes of advanced BC. Unlike other BC cases, patients with TNBC have little opportunity to choose targeted therapy; thus, chemotherapy remains the core treatment option. ${ }^{3,4}$ Generally, systemic chemotherapy has inevitable adverse effects and patients eventually show poor response due to rapidly acquired drug resistance and distant metastasis. ${ }^{5-7}$ Therefore, the development of specifically targeted and effective therapies to reduce the side effects in patients with TNBC remains an important clinical challenge. An innovative approach developed to overcome non-specificity in conventional cancer chemoprevention involves nanodrug delivery systems, which include liposomes, micelles, emulsions, and dendrimers, with passive or active targeting strategies. ${ }^{8-11}$

The cyclic arginylglycylaspartic acid (RGD) sequence, cyclo(Arg-Gly-Asp-d-Phe-Cys) (cRGD), is a "tumorhoming" cyclic peptide that binds directly to $\alpha \beta$ integrins. ${ }^{12}$ Among these integrins, $\alpha \mathrm{v} \beta 3$ is highly expressed on the surfaces of tumor and tumor-vessel cells in $\mathrm{BC}$ but not on healthy vessel cells. ${ }^{13,14}$ Surface conjugation of cRGD to nanoparticles (NPs) leads to the accumulation of the NPs in cancerous tissue, thus, sparing normal healthy cells to reduce adverse effects and improve therapeutic efficacy. ${ }^{15}$ The expression of the folate receptor alpha $(\mathrm{FR} \alpha)$ is significantly increased in patients with TNBC at both mRNA and protein levels and is considered a promising therapeutic target. ${ }^{16}$ FR $\alpha$ overexpression on cancer cell membranes may confer a growth advantage to tumors by satisfying the folate requirements of DNA synthesis in the rapidly-growing cells, while generally lower expression of folate receptor (FR) is found in most normal tissues. Folic acid (FA) is widely used as an active targeting ligand for functionalizing NPs. ${ }^{17-19}$

Monotherapy treatment is generally ineffective to completely destroy tumors due to heterogeneous distribution of drug and heterogeneity of cellular composition of certain cancer tissues. ${ }^{20-23}$ Thus, combination therapy, which targets and inhibits multiple essential pathways of tumor growth, invasiveness, and/or metastasis, holds great potential for enhancing therapeutic efficacy, lowering drug toxicity, and overcoming drug resistance. ${ }^{24,25}$ A rapid temperature increase above the threshold value of $42-45^{\circ} \mathrm{C}$ for $15-60$ $\min$ (or $>50^{\circ} \mathrm{C}$ for $4-6 \mathrm{~min}$ ) at local tumor sites can kill cancer cells. ${ }^{26}$ The selection of the external light source determines the effectiveness of the phototherapy. The process of light-sensitive materials absorbing NIR light and converting it to heat refers to photothermal therapy (PTT) ${ }^{27}$ NIR light does not affect the delivery system seriously with its low excitation energy, and can penetrate $10 \mathrm{~cm}$ deep for tumor excisional biopsies, which is much deeper than what can be achieved using ultraviolet (UV). Additionally, NIR is safer for humans than UV. ${ }^{28,29}$

Due to its high selectivity, low invasiveness, short recovery time, and safety for normal tissues, PTT is emerging as a powerful technique for the thermal destruction of cancerous cells. ${ }^{30}$ Therefore, the combination of chemotherapy and PTT in one system is considered a more effective approach for killing cancer cells.

Dopamine is a catecholamine that acts as an important neurotransmitter in the nervous system and has attracted considerable interest as a monomer of polydopamine (PDA), which self-assembles by oxypolymerization under alkaline conditions. ${ }^{31}$ PDA-based materials have been biomedically applied to biosensing, bioimaging, drug delivery, tissue engineering, and theragnostic and cancer photothermal treatment, particularly in the NIR-mediated PTT in vivo. ${ }^{32-35}$ Benefiting from the wide distribution of its monomer in humans, synthesized PDA is a biodegradable polymer that shows no obvious cytotoxicity in vivo or in vitro. ${ }^{32}$ In addition, the functional groups (catechol and amine groups) on the surface of PDA make it easy to modify. The abundant active groups on the surface of the PDA layer can graft some biomolecules with - $\mathrm{SH},-\mathrm{NH}_{2}$ and other groups through the Michael addition reaction or Schiff base reaction, to further functionalize the surface of the material and expand its application scope. ${ }^{36,37}$ As a result, PDA is an exceptional and particularly suitable PTT platform. PDA also exhibits broad absorption ranging from UV to NIR wavelengths. Due to its NIR region absorption and $40 \%$ photothermal conversion efficiency, which is much higher than those of previously reported for organic PTT agents, PDA is considered a highly effective and safe photothermal therapeutic agent for cancer treatment. ${ }^{33,38}$

A combination of chemotherapy drugs, including an anthracycline (such as epirubicin (EPI)), is recommended by the National Institute for Health and Care Excellence Guidelines (NICE, 2018). ${ }^{39}$ EPI-HCl, a 4'-epimer of doxorubicin, acts via DNA intercalation and inhibiting topoisomerase II, which further interferes with DNA, RNA, and protein synthesis. Compared with doxorubicin at equimolar doses, EPI shows similar efficacy and lower toxicity, and can be 
successfully incorporated into most standard chemotherapies for $\mathrm{BC}$ treatment. ${ }^{40}$ However, EPI shows cardiotoxicity when its cumulative dose exceeds $900 \mathrm{mg} / \mathrm{m}^{2.41-43}$ Therefore, the main objective of this study was to deliver and achieve the desired drug concentration at the intended site to minimize its toxicity.

In this study, we combined the photothermalchemotherapy effects of hybrid PDA and EPI by forming selfassembled NPs under electrostatic forces. First, L-cysteine was added to dopamine to form hybrid PDA-cysteine cores (PDAC cores) with reduced core sizes. Next, taking advantage of the positive charge of EPI at $\mathrm{pH} 7.0$, the electrostatic interactions of the negatively charged dimyristoyl phosphatidylglycerol (DMPG) and N-(methoxypolyethylene oxycarbonyl)-1,2-distearoryl-sn-glycero-3-phosphoethanolamine (DS PE-PEG)-coated PDAC cores and positively charged EPI were used to form EPI-loaded PDAC NPs (E/P-NPs). The E/ P-NPs were then further modified with lipid-PEG-cRGD and lipid-PEG-FA (E/PCF-NPs). The obtained E/PCF-NPs showed selective distribution in cancer cells rather than in normal cells. Under NIR laser irradiation, the EPI was released and combined with PDA photothermal treatment in the orthotopic mouse breast tumor model (Figure 1). The physicochemical properties, stability, and in vitro release of the developed NPs were characterized. In vitro cell studies were carried out to evaluate the combination of PTT and chemotherapeutic effects. Further, we evaluated toxicity, pharmacokinetic, and tissue distribution in vivo. Finally, the anti-tumor effects were evaluated in an orthotopic BC model.

\section{Materials and Methods}

\section{Materials}

Dopamine hydrochloride (purity $>98 \%$ ) was obtained from Shanghai Aladdin Biochemical Technology Co., Ltd. (Shanghai, China). EPI (purity $>99 \%$ ) was obtained from Zhejiang Haizheng Pharmaceutical Co., Ltd. (Zhejiang, China). L-cysteine acid (purity $>98 \%$ ) was obtained from Sigma-Aldrich (Shanghai) Trading Co., Ltd. (Shanghai, China). DMPG (purity >98\%), DSPE-PEG2000, and highly purified cholesterol were obtained from Shanghai Advanced Vehicle Technology Co., Ltd. (Shanghai, China). DSPEPEG2000-cRGD and DSPE-PEG2000-FA were from Xi'an Ruixi Biological Technology Co., Ltd. (Xi'an, China). Calcein $\mathrm{AM}$ and propidium iodide (PI) were purchased from Shanghai Shengsheng Biological Technology Co., Ltd. (Shanghai, China). Daunorubicin (purity $>98 \%$ ) was obtained from Shanghai Yuanye Bio-Technology Co., Ltd.

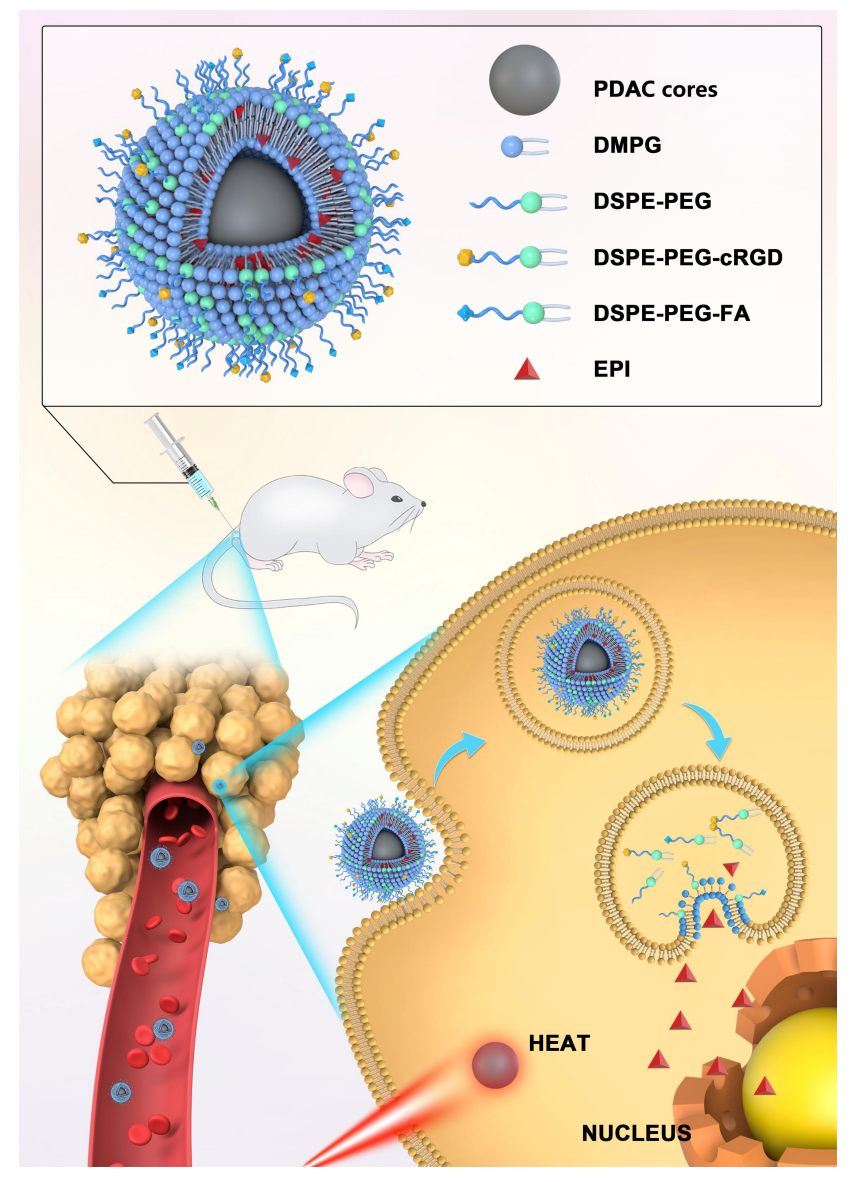

Figure I Schematic diagram of the structure and action mechanism of E/PCF-NPs.

(Shanghai, China). Antifade Mounting Medium with 40,6-diamidino-2-phenylindole (DAPI) was obtained from Vector Laboratories (CA, USA). All other chemicals were of analytical grade and were used as received.

\section{Cells}

Murine BC 4T1 cells were obtained from Beijing Dingguo Changsheng Biotechnology Co., Ltd. (Beijing, China). The 4T1 cells were cultured in RPMI 1640 (Beijing Suo Laibao Technology Co., Ltd., Beijing, China) containing 10\% fetal bovine serum (FBS; Thermo Fisher Scientific Co., Ltd. China), and penicillin-streptomycin solution $(100 \mathrm{U} / \mathrm{mL}$ each; Guangzhou Fansi Biotechnology Co., Ltd. Guangzhou, China). Cells were cultivated in a humidified incubator at $37^{\circ} \mathrm{C}$ and $5 \% \mathrm{CO}_{2}$ and harvested with $0.25 \%$ trypsin-EDTA before subculture.

\section{Animals}

Six-week-old female BALB/c mice were obtained from Beijing Weitong Lihua Experimental Animal Technology Co., Ltd. (Beijing, China). Female Sprague-Dawley rats 
$(200 \pm 20 \mathrm{~g})$ were provided by the Hunan SJA Laboratory Animals (Hunan, China). The animals were cared for at the animal experimental center at Jiangxi University of Traditional Chinese Medicine. The animal room was well ventilated and had a regular $12 \mathrm{~h}$ light-dark cycle throughout the experimental period. The animal experiments were performed according to study protocols approved by the Institutional Animal Care and Use Committee and the Laboratory Animal Welfare Ethics Committee of Jiangxi University of Traditional Chinese Medicine (Nanchang, China). Jiangxi University of Traditional Chinese Medicine Policy on Humane Care and Use of Laboratory Animals was strictly followed throughout the animal study.

\section{Preparation of the PDAC Cores}

PDA cores were prepared by oxidative self-polymerization in alkaline and aerobic environments. First, dopamine hydrochloride and L-cysteine amino acid (0.5 wt.\%, 3:2, $\mathrm{w} / \mathrm{w})$ were dissolved in distilled water at concentrations of 0.3 wt. $\%$ and $0.2 \%$, respectively, to form Solution A. Second, a mixture of $30 \%$ ethanol solution: $25 \%$ aqueous ammonia (26:1, v/v, pH 11.6) was prepared after stirring for $0.5 \mathrm{~h}$, to form Solution B. Solution A was added to Solution B drop wisely $(1: 13.5 \mathrm{v} / \mathrm{v})$. Subsequently, the mixture was stirred under dark for $24 \mathrm{~h}$ before dialyzing for $6 \mathrm{~h}$ using dialysis membrane (MWCO; $300 \mathrm{kDa}$ ) to obtain the PDAC cores. The PDAC cores were stored at $4^{\circ} \mathrm{C}$ in dark for further investigations. Their hydrodynamic diameter and zeta potential $(\zeta)$ were measured using a Zetasizer Nano ZS (ZS ZEN3690, Malvern, UK).

\section{Preparation of E/PCF-NPs}

DSPE-PEG2000-cRGD (74 nM) and DSPE-PEG2000-FA

(78 $\mathrm{nM})$ methanol solutions were mixed with $42 \mu \mathrm{M}$ DMPG and $3.4 \mu \mathrm{M}$ DSPE-PEG2000 methanol solution at a ratio of $1: 1000(\mathrm{v} / \mathrm{v})$, and $7.8 \mu \mathrm{M}$ EPI was dissolved in the mixture. The lipid and EPI solution were then added dropwise to the previously obtained PDAC core suspensions at a volume ratio of 1:3 with stirring, before dialyzing for $12 \mathrm{~h}$ to obtain E/PCF-NPs. PDAC cores modified with DMPG, DSPE-PEG2000, and DSPE-PEG2000cRGD loaded with EPI (E/PC-NPs) were prepared using the same protocol, without the addition of DSPE-PEG2000 -FA. PDAC cores modified with DMPG, DSPE-PEG2000, and DSPE-PEG2000-FA loaded with EPI (E/PF-NPs) were prepared using the same protocol, without the addition of DSPE-PEG2000-cRGD.
Solutions of DMPG $(42 \mu \mathrm{M})$ and DSPE-PEG2000 methanol solution $(3.4 \mu \mathrm{M})$ were prepared. The lipid solution was then added drop-wise to the previously obtained PDAC core suspensions at a volume ratio of $1: 3$ with stirring, followed by dialysis for $12 \mathrm{~h}$. The PDAC cores modified with DMPG and DSPE-PEG2000, without the addition of EPI and modification of DSPE-PEG2000-cRGD and DSPEPEG2000-FA, were named as PDA-NPs. PDAC cores modified with DMPG and DSPE-PEG2000 loaded with EPI (E/ P-NPs) were prepared using the same protocol, except that the lipid solution without DSPE-PEG2000-cRGD and DSPE-PEG2000-FA, was used to dissolve EPI to achieve the concentration of $7.8 \mu \mathrm{M}$ before adding to PDAC core suspensions. The obtained NPs were stored at $4{ }^{\circ} \mathrm{C}$ until further analysis. The abbreviations and full names of all preparations are listed in Table S1.

\section{The Contents of cRGD and FA on the Surface of Nanoparticles}

The contents of cRGD on the surfaces of E/PC-NPs and E/ PCF-NPs were determined by an enzyme-linked immunosorbent assay (ELISA) kit as recommended by the manufacturer's instructions (JL49363, Jiangsu Jianglai Biotechnology Co., Ltd, Jiangsu, China). The contents of FA on the surfaces of E/PF-NPs and E/PCF-NPs were determined by a chemiluminescence kit as recommended by the manufacturer's instructions (2012-2,400,059, Bioscience (Tianjin) Bio-technology Co., Ltd, Tianjin, China).

\section{Temperature Elevation Induced by Laser Irradiation}

First, $1 \mathrm{~mL}$ samples of different concentrations of PDA-NPs $(50,100,200$, and $300 \mu \mathrm{g} / \mathrm{mL})$ were irradiated with an 808 $\mathrm{nm}$ laser with a power density of $2.15 \mathrm{~W} / \mathrm{cm}^{2}$. The temperature of the solutions was monitored using a TES-1310 thermocouple thermometer (Suzhou Runqi Electronic Technology, Suzhou, China) submerged in the solution every $30 \mathrm{~s}$.

\section{Thermal Toxicity}

$4 \mathrm{~T} 1$ cells were seeded in $35 \mathrm{~mm}$ cell culture dishes with glass bottoms at a density of $1 \times 10^{4}$ per dish and incubated for $24 \mathrm{~h}$ before treatment. The cell culture medium was removed and replenished with culture medium containing PDA-NPs $(200 \mu \mathrm{g} / \mathrm{mL})$ for $2 \mathrm{~h}$. Cells were exposed to a NIR laser $\left(808 \mathrm{~nm}, 2.15 \mathrm{~W} / \mathrm{cm}^{2}\right)$ for $10 \mathrm{~min}$ then washed with phosphate-buffered saline (PBS) three times and 
stained with calcein AM and PI according to the manufacturer's instructions (Beijing Solarbio Science \& Technology Co., Ltd, Beijing, China). Finally, the alive cells (green) and dead cells (red) were visualized using an Eclipse Ti-U Inverted Fluorescence Microscope at 10× magnification (Nikon, Japan).

The viability of cells treated with PDA-NPs with or without irradiation was also determined using a (3-(4,5-dimethylthiazol-2-yl)-2,5-diphenyltetrazolium bromide) (MTT) assay. Murine 4T1 cells were seeded in 96well plates at a density of 10,000 cells per well in RPMI1640 complete medium and incubated at $37^{\circ} \mathrm{C}$ in $5 \% \mathrm{CO}_{2}$ overnight. Complete medium with and without cells was used as the control and blank groups, respectively. To evaluate the cytotoxicity of the PDA-NPs, the culture media were replaced with $100 \mu \mathrm{L}$ of media with $10 \%$ FBS containing a series of PDA concentrations $(30,60,120,240,480$, and $960 \mu \mathrm{g} / \mathrm{mL}$ ) or free EPI (25 and $200 \mu \mathrm{g} / \mathrm{mL})$. The cells were cultured for $2 \mathrm{~h}$ and exposed to NIR laser irradiation (808 nm, $2.15 \mathrm{~W} / \mathrm{cm}^{2}$ ) for $3 \mathrm{~min}$. Subsequently, $20 \mu \mathrm{L}$ of MTT solution $(5.0 \mathrm{mg} / \mathrm{mL})$ was added to each well, and the cells were further incubated for $4 \mathrm{~h}$ at $37^{\circ} \mathrm{C}$. Next, the culture medium was removed, and replaced with $150 \mu \mathrm{L}$ of dimethyl sulfoxide to dissolve the formazan crystals formed by the living cells. The absorption was recorded at $490 \mathrm{~nm}$ using an $\mathrm{i} 3 \mathrm{x}$ microplate reader (SpectraMax, Molecular Devices, CA, USA). The cell viability for each group was calculated and normalized to the untreated control cells $(n=6)$.

\section{Characterization of E/PCF-NPs}

The morphology of the PDA-NPs and E/PCF-NPs was examined using transmission electron microscopy (TEM) (Tecnai G 2 Spirit TWIN $120 \mathrm{kV}$, FEI, USA), atomic force microscopy (AFM) (SPM9700, SHIMADZU, Japan), and scanning electron microscopy (SEM) (Quanta250, FEI, USA). Their hydrodynamic diameter and zeta potential

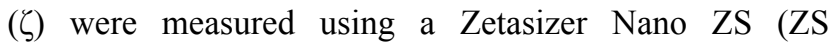
ZEN3690, Malvern, UK).

The drug loading (DLwt.\%) and entrapment efficiency $(\mathrm{EE} \%)$ of EPI were determined using an ultrafiltration method for separating the non-entrapped drug from the NPs. ${ }^{44}$ Briefly, $300 \mu \mathrm{L}$ of NPs were placed in an ultrafiltration tube (Nanosep MF; Pall Corporation, Port Washington, NY, USA) fitted with a filter membrane (MWCO: $30 \mathrm{kDa}$ ). Free drug in the solution was collected by centrifugation at $10,000 \mathrm{rpm}$ for $15 \mathrm{~min}$ using a highspeed refrigerated centrifuge (HC-3018R; Anhui USTC
Zonkia Scientific Instruments Co., Ltd, Anhui, China). The EPI content of the ultrafiltrate $\left(\mathrm{C}_{\text {free }}\right)$ was determined using high-performance liquid chromatography (HPLC) on a Diamonsil C18 column $(250 \times 4.6 \mathrm{~mm}, 5 \mu \mathrm{m}$; Beijing Dikma Technologies Co, Ltd., Beijing, China) at $254 \mathrm{~nm}$. The mobile phase was a mixture of phase A and B (57:43, v/v). Phase A was a mixture of acetonitrile and methanol $(6: 1, \mathrm{v} / \mathrm{v})$. Phase B was $0.02 \mathrm{M}$ phosphoric acid solution containing $0.29 \%$ sodium dodecyl sulfate, and the flow rate was $1.0 \mathrm{~mL} / \mathrm{min}$. The column temperature was maintained at $30^{\circ} \mathrm{C}$, and the injection volume was $10 \mu \mathrm{L}$. The NP samples $(0.1 \mathrm{~mL})$ were diluted with $2.0 \mathrm{~mL}$ of methanol and filtered using a $0.22 \mu \mathrm{m}$ film to determine the total drug concentration $\left(\mathrm{C}_{\text {total }}\right)$ by HPLC. The preparations were lyophilized for $48 \mathrm{~h}$ and the mass of the NPs was determined. ${ }^{45}$ EE\% and DLwt.\% were determined using Equations (1) and (2):

$$
\mathrm{EE} \%=\left(\mathrm{C}_{\text {total }}-\mathrm{C}_{\text {free }}\right) / \mathrm{C}_{\text {total }} \times 100
$$

DLwt. $\%=\left(\mathrm{C}_{\text {total }}-\mathrm{C}_{\text {free }}\right) /$ dried nanoparticles $\times 100$

\section{Stability}

The size and distribution of the NPs were measured at various time intervals $(0,24,48,96,120$, and $168 \mathrm{~h})$ to evaluate storage stability at $4^{\circ} \mathrm{C}$.

Meanwhile, the size distribution of NPs was determined after incubation with RPMI-1640 complete medium containing $10 \%$ FBS to mimic their stability in vivo. NPs and culture medium were mixed at a ratio of 1:6.4 (v/v), ${ }^{46}$ and the samples were withdrawn and analyzed at scheduled time points.

\section{In vitro Release Under NIR Excitation}

Investigation of the EPI release profile was conducted using a SOTAX AT7 smart dissolution apparatus (Sotax, Horsham, PA, USA) packed with glass beads $(1 \mathrm{~mm})$ in closed-system mode at $37^{\circ} \mathrm{C}$. The NPs were placed in cells using $100 \mathrm{~mL}$ of PBS at $\mathrm{pH} 7.4,6.5$, and 5.0 (without or after irradiation at $808 \mathrm{~nm}$ and $2.15 \mathrm{~W} / \mathrm{cm}^{2}$ for $5 \mathrm{~min}$ ) at a flow rate of $4 \mathrm{~mL} / \mathrm{min}$ to mimic the $\mathrm{pH}$ environment in the blood, tumor microenvironment, and lysate. ${ }^{47}$ Samples (1 $\mathrm{mL})$ were withdrawn and replenished with an equal volume of fresh blank medium. Samples were filtered through a $0.22 \mu \mathrm{m}$ film and the EPI content of the medium was determined using the HPLC conditions described previously. 


\section{Cell Viability Assay}

The chemo-cytotoxicity effect of the E/PCF-NPs was analyzed using an MTT assay as described previously. The cells were incubated with different concentrations of free EPI (Free EPI), E/PC-NPs, E/PF-NPs, and E/PCF-NPs for $24 \mathrm{~h}$. The cell viability for each group was calculated and normalized to the untreated cell control $(n=6)$. The mean drug concentration for $50 \%$ inhibition of cell growth (IC50) was calculated.

The effect of E/PCF-NPs combined with NIR laser on 4T1 cells was also determined as previously described. ${ }^{9}$ The cells were treated with the E/PCF-NPs (PDA: $200 \mu \mathrm{g} /$ $\mathrm{mL}$; EPI: $25 \mu \mathrm{g} / \mathrm{mL}$ ) for $2 \mathrm{~h}$ and exposed to a NIR laser $\left(808 \mathrm{~nm}, 2.15 \mathrm{~W} / \mathrm{cm}^{2}\right)$ for $3 \mathrm{~min}$. The cell viability was measured by MTT assay and normalized to the untreated control cells $(n=6)$.

\section{Detection of Reactive Oxygen Species (ROS)}

Fluorescent probe 2',7'-dichlorofluorescein diacetate (DCFH-DA) was used for active oxygen detection. DCFHDA has no fluorescence per se; after entering the cell, it is hydrolyzed by intracellular esterase to generate DCFH. ROS in the cells oxidize non-fluorescent DCFH to generate fluorescent dichlorofluorescein (DCF). The fluorescence indicates the level of ROS in the cell. ${ }^{48} 4 \mathrm{~T} 1$ cells $\left(1 \times 10^{6}\right.$ per well $)$ were seeded on a 6 -well cell culture plate and incubated at $37^{\circ} \mathrm{C}$ in a $5 \% \mathrm{CO}_{2}$ cell incubator for $24 \mathrm{~h}$. Then, the medium was removed, and the cells were washed three times with PBS. DCFH-DA probe $(2 \mathrm{~mL})$ was added to each well and the samples were incubated in an incubator at $37^{\circ} \mathrm{C}$ for $20 \mathrm{~min}$. Following incubation, the cells were washed three times with RPMI 1640 medium for 2 min each to completely remove the DCFH-DA that had not entered the cells. After washing, $2 \mathrm{~mL}$ of EPI solution, PDA-NPs, and E/PCF-NPs (PDA: $200 \mu \mathrm{g} / \mathrm{mL}$; EPI: $25 \mu \mathrm{g} / \mathrm{mL}$ ) suspensions were added to each well. After incubation for $1 \mathrm{~h}$, each group was subjected to photothermal treatment (NIR laser, $808 \mathrm{~nm}, 2.15 \mathrm{~W} / \mathrm{cm}^{2}$ ) for $10 \mathrm{~min}$. After washing with medium three times, the fluorescence was measured using a fluorescence microplate reader at an excitation wavelength of $488 \mathrm{~nm}$ and emission wavelength of $525 \mathrm{~nm}$ following the manufacturer's instructions (Beyotime Biotechnology Co., Ltd, Shanghai, China).

\section{Detection of Oxidized Nicotinamide}

Adenine Dinucleotide/Reduced Nicotinamide Adenine Dinucleotide $\left(\mathrm{NAD}^{+} / \mathrm{NADH}\right)$

NAD is a coenzyme found in all cells as either the oxidized $\left(\mathrm{NAD}^{+}\right)$or reduced $(\mathrm{NADH})$ form. $\mathrm{NAD}^{+}$ plays an important role in cells both in vitro and in vivo, and the reduction of $\mathrm{NAD}^{+}$is one of the main factors contributing toward cell death. ${ }^{49}$ Cells $\left(1 \times 10^{6}\right.$ per well) were inoculated in 6-well plates, and the Free EPI, PDA-NPs and E/PCF-NPs (PDA: $200 \mu \mathrm{g} / \mathrm{mL}$; EPI: $25 \mu \mathrm{g} / \mathrm{mL}$ ) were added and incubated for $1 \mathrm{~h}$; blank controls were maintained simultaneously. Next, each group was subjected to photothermal treatment (NIR laser, 808 $\mathrm{nm}, 2.15 \mathrm{~W} / \mathrm{cm}^{2}$ ) for $10 \mathrm{~min}$. The culture solution was aspirated, and the cells were washed three times with PBS before $2 \mathrm{~mL}$ of $\mathrm{NAD}^{+} / \mathrm{NADH}$ extract was added, and the solution was gently mixed to promote cell lysis. The extract was centrifuged at $12,000 \times \mathrm{g}$ and $4^{\circ} \mathrm{C}$ for 10 min, and the supernatant was collected for testing. First, $20 \mu \mathrm{L}$ of the sample to be tested was added to a 96-well plate, and three replicates were established in each group. Next, $90 \mu \mathrm{L}$ of ethanol dehydrogenase solution was added to each well, and samples were incubated at $37^{\circ} \mathrm{C}$ for $10 \mathrm{~min}$ in dark. Then, $10 \mu \mathrm{L}$ of coloring solution was added to the samples and mixed well. The solution was incubated for $30 \mathrm{~min}$ in dark at $37^{\circ} \mathrm{C}$, and the absorbance was measured at $450 \mathrm{~nm}$ as recommended by the manufacturer (Beyotime Biotechnology Co., Ltd., Shanghai, China).

\section{Apoptosis Assays}

Based on the results of the MTT assays, 4T1 cells $\left(1 \times 10^{6}\right.$ per well) were divided into eight groups: control, Free EPI, PDA-NPs, E/P-NPs, E/PC-NPs, E/PF-NPs, and E/ PCF-NPs with or without NIR laser irradiation (PDA: $200 \mu \mathrm{g} / \mathrm{mL}$; EPI: $25 \mu \mathrm{g} / \mathrm{mL}$ ). After incubation for $1 \mathrm{~h}$, cells were exposed to the NIR laser $(808 \mathrm{~nm}, 2.15 \mathrm{~W} /$ $\mathrm{cm}^{2}$ ) according to the experimental design, for $10 \mathrm{~min}$. Next, the cells were collected, washed with PBS, and resuspended in $200 \mu \mathrm{L}$ of staining buffer. All samples were stained using annexin V/PI according to the manufacturer's protocol (Beijing Ruibang Xingye Technology Co., Ltd., Beijing, China) and subjected to flow cytometry within $1 \mathrm{~h}$ (BD Gallios, BD Biosciences, CA, US). Data were analyzed using FlowJo 8.6 software (TreeStar). 


\section{Cellular Uptake}

4T1 cells were seeded in $35 \mathrm{~mm}$ cell culture dishes with glass bottoms at a density of $1 \times 10^{6}$ per dish and incubated for $24 \mathrm{~h}$ before treatment. Free EPI, PDA-NPs, E/ P-NPs, E/PC-NPs, E/PF-NPs, and E/PCF-NPs (EPI concentration $1 \mu \mathrm{g} / \mathrm{mL}$ ) were added to the dishes. The cells were then cultured for another 4 or $12 \mathrm{~h}$ at $37^{\circ} \mathrm{C}$. After washing three times, the cells were fixed with $4 \%$ paraformaldehyde. After fixation, DAPI mounting medium was added for nuclear staining. The cellular uptake of the NPs was observed using an SP8 confocal microscope (Leica, Germany) and quantified using ImageJ software (National Institute of Health).

The extent of cellular uptake was further investigated by flow cytometry. The cells were seeded into six-well plates at a density of $1 \times 10^{6}$ cells/well. Culture media containing Free EPI, PDA-NPs, E/P-NPs, E/PC-NPs, E/ PF-NPs, and E/PCF-NPs (EPI $1 \mu \mathrm{g} / \mathrm{mL}$ ) were used to incubate cells for 4 and $12 \mathrm{~h}$. Next, the cells were collected, washed, and resuspended in $0.5 \mathrm{~mL}$ cold PBS on ice for measurements using a flow cytometer (BD Gallios, BD Biosciences, CA, USA). Data were analyzed using FlowJo 8.6 software (TreeStar).

\section{Pharmacokinetic Study}

First, 15 healthy Sprague-Dawley rats were randomly divided into five groups and fasted overnight before the experiment. Free EPI, E/P-NPs, E/PC-NPs, E/PF-NPs, and E/PCF-NPs were intravenously administered at a dose of $10 \mathrm{mg} / \mathrm{kg}$. Blood samples $(500 \mu \mathrm{L})$ were withdrawn from the retro-orbital plexus at scheduled time points after injection $(0.017,0.05,0.083,0.25,0.5,1,2,4,8,12,24$, 48 , and $72 \mathrm{~h}$ ). The blood samples were centrifuged at 2300 $\times \mathrm{g}$ for $5 \mathrm{~min}$ at $25^{\circ} \mathrm{C}$. Then, $100 \mu \mathrm{L}$ of the separated plasma was mixed with $100 \mu \mathrm{L}$ of the internal standard daunorubicin $(1 \mu \mathrm{g} / \mathrm{mL})$ before the addition of methanol $(600 \mu \mathrm{L})$ to precipitate proteins. After centrifugation (10,000 rpm, $5 \mathrm{~min}$ ), the supernatant was dried under $\mathrm{N}_{2}$ and the EPI was dissolved in methanol and determined using an ultra-high performance liquid chromatography/ mass spectrometry system (UHPLC/MS).

The measurement was carried out on a UHPLC/MS system using a TRIPLE QUDA 4500 liquid chromatograph triple quadrupole mass spectrometer equipped with an electrospray ion source in positive mode (AB SCIEX, Framingham, MA, USA). Chromatographic separation was achieved on a Welch Ultimate UHPLC C18 column
$(50 \times 2.1 \mathrm{~mm}, 1.8 \mu \mathrm{m})$. The column and autosampler tray were maintained at $25^{\circ} \mathrm{C}$ and $4^{\circ} \mathrm{C}$, respectively. The mobile phase used was a mixture of solvent A $(0.3 \%$ formic acid in water) and B (acetonitrile). The gradient program used was as follows: initial Phase $0-1.0 \mathrm{~min}$, a linear change to $A: B(95: 5, \mathrm{v}: \mathrm{v}) ; 1.0-1.8 \mathrm{~min}$, a linear change to A:B (40:60, v:v); 1.8-2.6 min, a linear change to $\mathrm{A}: \mathrm{B}(5: 95, \mathrm{v}: \mathrm{v})$, where it was held for $0.2 \mathrm{~min} ; 2.8-4.0$ min, linear change to A:B (95:5, v:v), where it was held until the end of the $6 \mathrm{~min}$ run. The flow rate was maintained at $0.3 \mathrm{~mL} / \mathrm{min}$. The injection volume was $5 \mu \mathrm{L}$.

The analytes were ionized by an electrospray ionization source in positive ion mode under the following source conditions: nitrogen for protection; flow rate $9 \mathrm{~L} /$ min; dry gas temperature $350^{\circ} \mathrm{C}$; sprayer pressure $50 \mathrm{psi}$; capillary voltage $4000 \mathrm{~V}$; ion collection mode MRM; EPI $\mathrm{m} / \mathrm{z}$ : 544/130.1; DP positive ion mode optimized cluster voltage: $120 \mathrm{eV}$; CE impact voltage: $13 \mathrm{~V}$; daunorubicin hydrochloric acid $\mathrm{m} / \mathrm{z}: 528.2 / 321.1$; DP positive ion mode optimized cluster voltage: $100 \mathrm{eV}$; CE impact voltage: $25 \mathrm{~V}$. The internal standard ratio was calculated by dividing the analyte peak area by the peak area of the internal standard. Standard curves for EPI were determined by plotting the internal standard ratio versus the concentration of analyte in each sample. The maximum plasma concentration $\left(\mathrm{C}_{\max }\right)$, area under the concentration-time curve from zero to the final time point $\left(\mathrm{AUC}_{0 \rightarrow \mathrm{t}}\right)$, mean residence time from zero to the last time point $\left(\mathrm{MRT}_{0 \rightarrow \mathrm{t}}\right)$, apparent volume of distribution $\left(\mathrm{V}_{\mathrm{z}}\right)$, and total plasma clearance $(\mathrm{CL})$ were calculated by PK solver software.

\section{Tissue Distribution of Nanoparticles}

Six-week-old healthy female BALB/c mice were inoculated with $50 \mu \mathrm{L}$ of $4 \mathrm{~T} 1\left(1 \times 10^{7}\right.$ cells per mouse $)$ in the mammary gland to establish the orthotopic $\mathrm{BC}$ model. The $\mathrm{BALB} / \mathrm{c}$ mice bearing $400 \mathrm{~mm}^{2}$ tumors were divided into five groups of nine mice each. Free EPI, E/P-NPs, E/PCNPs, E/PF-NPs, and E/PCF-NPs were intravenously administered at a dose of $10 \mathrm{mg} / \mathrm{kg}$. Three mice were euthanized without treatment, and their tissues were used as blank controls and for the preparation of spiked control samples.

The mice were euthanized in groups of three at 1,6 , and $24 \mathrm{~h}$, and heart, liver, spleen, lungs, kidneys, brain, and tumor tissues were collected and weighed. Tissue samples were homogenized with $0.2 \mathrm{mg} / \mathrm{mL}$ saline, and $1 \mu \mathrm{g} / \mathrm{mL}$ of daunorubicin was added as an internal standard. Methanol 
solution was added to the mixture $(3: 1, \mathrm{v} / \mathrm{v})$ and the tissue samples were vortexed followed by centrifuged at $10,000 \mathrm{rpm}$ for $5 \mathrm{~min}$ at $4^{\circ} \mathrm{C}$. The supernatants were dried under nitrogen and redissolved in methanol for UHPLC/MS measurement, as described earlier.

\section{Orthotopic Breast Cancer Model}

Six-week-old healthy female BALB/c mice were inoculated with $50 \mu \mathrm{L}$ of $4 \mathrm{~T} 1\left(1 \times 10^{7}\right.$ cells per mouse $)$ in the mammary gland to establish the orthotopic $\mathrm{BC}$ model. The tumor growth of each mouse was monitored every two days and calculated using formula (3):

$$
\mathrm{V}=\left(\mathrm{L} \times \mathrm{W}^{2}\right) / 2
$$

where $\mathrm{V}$ is the tumor volume, $\mathrm{L}$ is the larger perpendicular diameter, and $\mathrm{W}$ is the smaller perpendicular diameter. Tumor was weighed at the end of the experiment and imaged.

\section{Evaluation of Therapeutic Efficacy for Early-Stage Tumor Progression}

The anti-tumor efficacy of the NPs was evaluated in breast tumor-bearing mice. When the tumor volume reached $150 \mathrm{~mm}^{3}$ (designated Day 0), the mice were randomly divided into 14 groups of five mice each. Saline, Saline and NIR, PDA-NPs, PDA-NPs and NIR, Free EPI, Free EPI and NIR, E/P-NPs, E/P-NPs and NIR, E/PC-NPs, E/ PC-NPs and NIR, E/PF-NPs, E/PF-NPs and NIR, E/PCFNPs, and E/PCF-NPs and NIR were intravenously injected into mice three times at seven-day intervals (Day 0, 7, and 14) at an EPI dose of $5 \mathrm{mg} / \mathrm{kg}$. One hour after the injection, the tumors of the NIR treatment groups were irradiated $\left(808 \mathrm{~nm}, 2.15 \mathrm{~W} / \mathrm{cm}^{2}\right)$ for $5 \mathrm{~min}$. The overall body weights of each group were also monitored.

\section{Hematoxylin and Eosin (H\&E) Staining}

Hearts, livers, spleens, lungs, kidneys, brains, and tumors were collected from each group and stained using a H\&E staining kit (Shanghai Bioengineering Co., Ltd., Shanghai, China) and observed using an Eclipse NI-U fluorescence microscope (Nikon Corporation, Tokyo, Japan) to investigate the NP toxicity.

\section{Evaluation of Therapeutic Efficacy for Late-Stage Tumor Progression}

Tumor-bearing mice with $500 \mathrm{~mm}^{3}$ tumors were treated with E/P-NPs and E/P-NPs+NIR via tail vein injection three times at seven-day intervals (Day 0, 7, and 14) at an EPI dose of $5 \mathrm{mg} / \mathrm{kg}$. One hour after the injection, the tumors in the NIR treatment groups were locally irradiated with the $808 \mathrm{~nm}$ laser, $2.15 \mathrm{~W} / \mathrm{cm}^{2}$ for $5 \mathrm{~min}$. The tumors were measured with a Vernier caliper and the tumor volume was calculated as described above.

\section{Terminal Deoxynucleotidyl Transferase-Mediated Nick End Labeling (TUNEL) Assay}

First, $24 \mathrm{~h}$ after the initial injection of E/PCF-NPs and NIR irradiation, the tumors of each group were collected and fixed in $4 \%$ formalin, paraffin-embedded, and sectioned into $5 \mu \mathrm{m}$ samples for the TUNEL assay, as recommended by the manufacturer (Beyotime Biotechnology Co., Ltd, Shanghai, China). DAPI mounting medium was added for cell nuclei staining using an Eclipse Ti-U Inverted Fluorescence Microscope at $10 \times$ magnification (Nikon, Japan). The apoptotic index was calculated by dividing the number of TUNEL-positive cells by the total number of cells in the field. Finally, 10 randomly selected microscope fields were analyzed quantitatively using ImageJ software (US National Institutes of Health).

\section{Statistics}

Results are expressed as a mean \pm standard deviation (SD). Statistical analysis was conducted using one-way analysis of variance and Student's $t$-test. Results with $p$-values $<0.05$ were statistically significant.

\section{Results and Discussion \\ Preparation and Characterization of PDAC Cores}

PDA particles were formed by polymerization of a dopamine monomer at high $\mathrm{pH},{ }^{50}$ which evolved via the addition of a monomer or small oligomers to the chain. The small oligomer chains rapidly aggregated to form entities that gradually formed the larger structures. The size of PDA particles varies with the dopamine concentration and buffer. ${ }^{51}$ Here, we used ammonia to achieve alkaline conditions. L-cysteine was selected to participate in the aggregation of the dopamine monomers. The effect of a series of amino acids (L-cysteine, histidine, lysine, and arginine) on the aggregation process was investigated (See details in Table S2) and we found that only L-cysteine and dopamine alone generated small, welldistributed particles (L-cysteine and dopamine formed 
particles: $88.8 \pm 8.4 \mathrm{~nm}, \mathrm{PDI}=0.076 \pm 0.01)$. The enhancement in size distribution resulting from L-cysteine addition may be derived from the positive charges of the amino groups, which interact with the moieties of dopamine. The aggregation of dopamine might be delayed due to the L-cysteine, which would enable obtaining smaller particles at the same time.

\section{Preparation and Characterization of E/ PCF-NPs}

The smart E/PCF-NPs were prepared using the methanolinjection method followed by dialysis. The obtained PDAC cores were coated with negatively charged lipids by inner hydrogen bond or electrostatic interaction, while the hydrophilic heads of DMPG, DSPE-PEG-FA, and DSPE-PEG-cRGD were also able to attract positively charged EPI hydrochloride at $\mathrm{pH}$ 7.0. PDAC cores were modified with DMPG and DSPE-PEG2000 without the addition of EPI to form PDA-NPs. PDAC cores were modified with DMPG and DSPE-PEG2000 loaded with EPI to form E/P-NPs. PDAC cores were modified with DMPG, DSPE-PEG2000, and DSPE-PEG2000-cRGD and loaded with EPI to form E/PC-NPs. PDAC cores were modified with DMPG, DSPE-PEG2000, and DSPEPEG2000-FA and loaded with EPI to form E/PF-NPs. The contents of cRGD and FA on the surface of E/PFNPs were $18.5 \mathrm{nM}$ and $19.5 \mathrm{nM}$, respectively.

The photothermal effects of different concentrations of PDA-NPs under $808 \mathrm{~nm}$ laser irradiation were tested using water as a negative control. Figure $2 \mathrm{~A} 1$ and $\mathrm{A} 2$ shows the concentration-dependent photothermal effect. The higher the concentration, the more rapid the temperature increase. For the PDA-NPs at $200 \mu \mathrm{g} / \mathrm{mL}$, the temperature increased to $45.7^{\circ} \mathrm{C}$ in $5 \mathrm{~min}$, and to $54.8^{\circ} \mathrm{C}$ in $10 \mathrm{~min}$, which leads to hyperthermia and cell death by thermolysis in tumor cells; compared with the cancerous cells, normal cells were more heat tolerant. ${ }^{52-54}$

Figure 2A3 shows the photothermal conversion efficiency of the PDA-NPs $(200 \mu \mathrm{g} / \mathrm{mL})$ did not decrease after five repeated irradiation periods, indicating that PDA-NPs containing hybrid PDAC cores and coated with lipids retain good photothermal conversion efficiency, as with polydopamine alone reported previously. ${ }^{33}$ Therefore, we used PDA-NPs at $200 \mu \mathrm{g} / \mathrm{mL}$ in the subsequent experiments.

The cells were incubated with PDA-NPs for $30 \mathrm{~min}$, then exposed to an $808 \mathrm{~nm}$ laser at $2.15 \mathrm{~W} / \mathrm{cm}^{2}$ for $10 \mathrm{~min}$.
After treatment, the cells were stained with both calcein AM and PI. As shown in Figure 2A4, under excitation, a clear demarcation line between regions of live (green) and dead (red) cells was observed for 4T1 cells, and most cells subjected to laser irradiation were killed. In contrast, cells treated with PDA-NPs alone without NIR showed negligible cell death. These results suggest that PDA-NPs killed cancer cells effectively through the photothermal effect induced by NIR irradiation, which is consistent with the result of a previous study on PDA. ${ }^{33}$ We further evaluated the photothermal cytotoxicity of the PDA-NPs on cancer cells quantitatively using an MTT assay (Figure 2B). After incubating 4T1 cells with PDA-NPs at a series of concentrations, neither the cell viability nor proliferation were affected in absence of laser radiation. Even at the highest tested dose of PDA $(960 \mu \mathrm{g} / \mathrm{mL})$, cell viability remained approximately 90\% $(p<0.0001)$. However, upon laser irradiation, 4T1 cell viability decreased significantly, and less than $30 \%$ of cells remained alive for the PDA-NPs at a concentration of $200 \mu \mathrm{g} / \mathrm{mL}$. The EPI toxicity on $4 \mathrm{~T} 1$ cells, with and without irradiation, did not change significantly at either tested concentrations (25 or $200 \mu \mathrm{g} / \mathrm{mL}$ ).

Since PDA particles are characterized by high efficiency of photothermal conversion and high biocompatibility, ${ }^{33}$ they are promising for future therapeutics relating to cancer prevention. However, certain disadvantages of PDA particles limit their usage. First, they could be recognized and phagocytized by the reticuloendothelial system (RES). ${ }^{55}$ Second, they do not have targeting properties and cannot be enriched in cancer tissues. The data in Figure 2 demonstrate that PDA-NPs could preserve the good photothermal conversion efficiency, which results from the photothermal effect under NIR irradiation and would not cause cell death in normal cells without NIR irradiation.

The size and morphology of the particles were critical to achieve passive targeting of the tumor site through the enhanced permeability and retention effect (EPR). ${ }^{56}$ The TEM, AFM and SEM of E/PCF-NPs and PDA-NPs are shown in Figure 3A. Their hydrodynamic diameter and zeta potential $(\zeta)$, size, zeta potential $(\mathrm{mV})$, DLwt.\% and $\mathrm{EE} \%$ are summarized in Figure 3B. As shown in Figure 2A, SEM of the PDA-NPs showed particle size of approximately $100 \mathrm{~nm}$ with good dispersion, and they were spherical or quasi spherical in shape. Coating with DSPE-PEG-cRGD and DSPE-PEG-FA led to an increase in E/PCF-NP size. Due to the interaction between EPI and DMPG, the EE\% of EPI increased to $99 \%$. Modification with DSPE-PEG-cRGD 
A
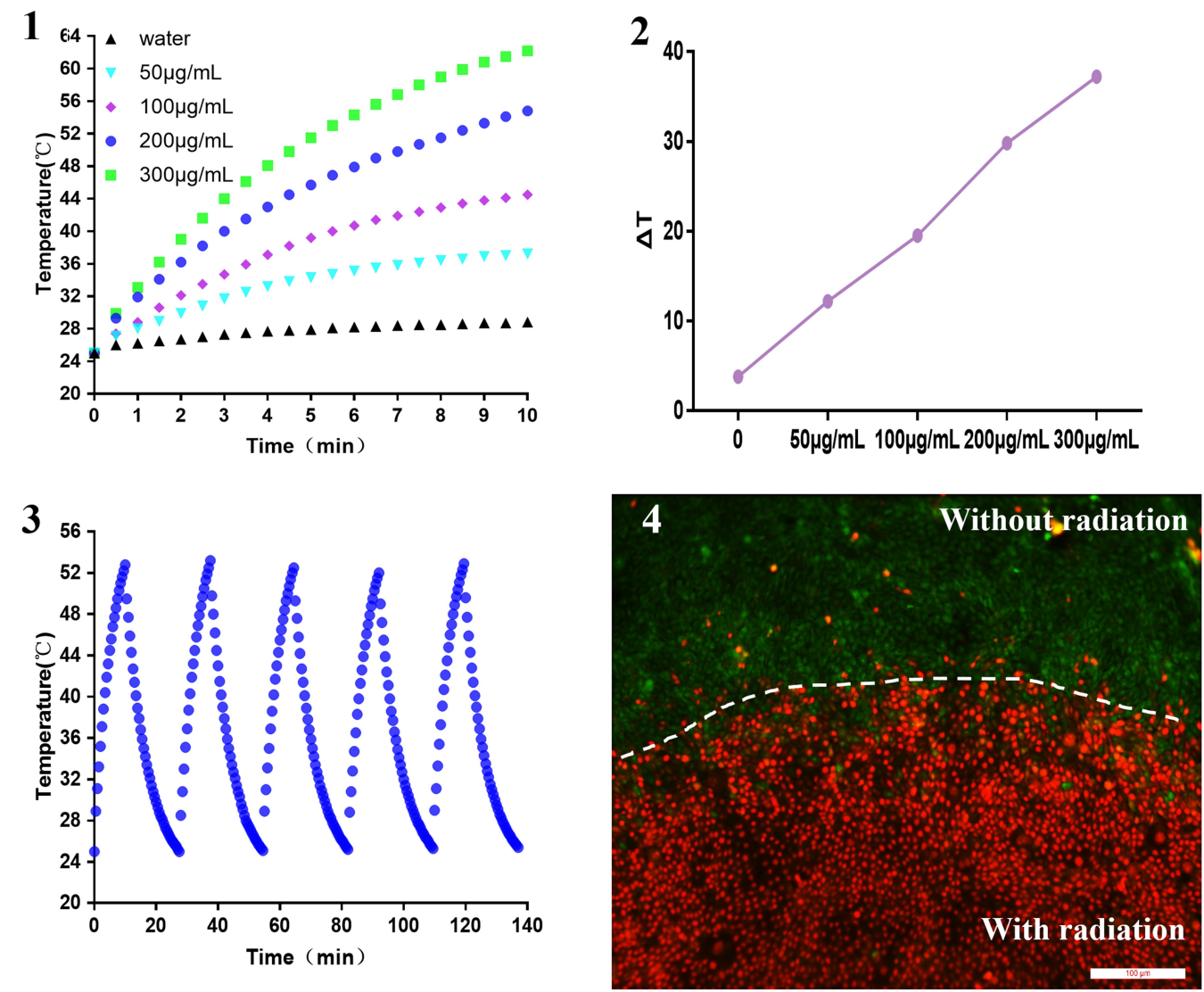

B

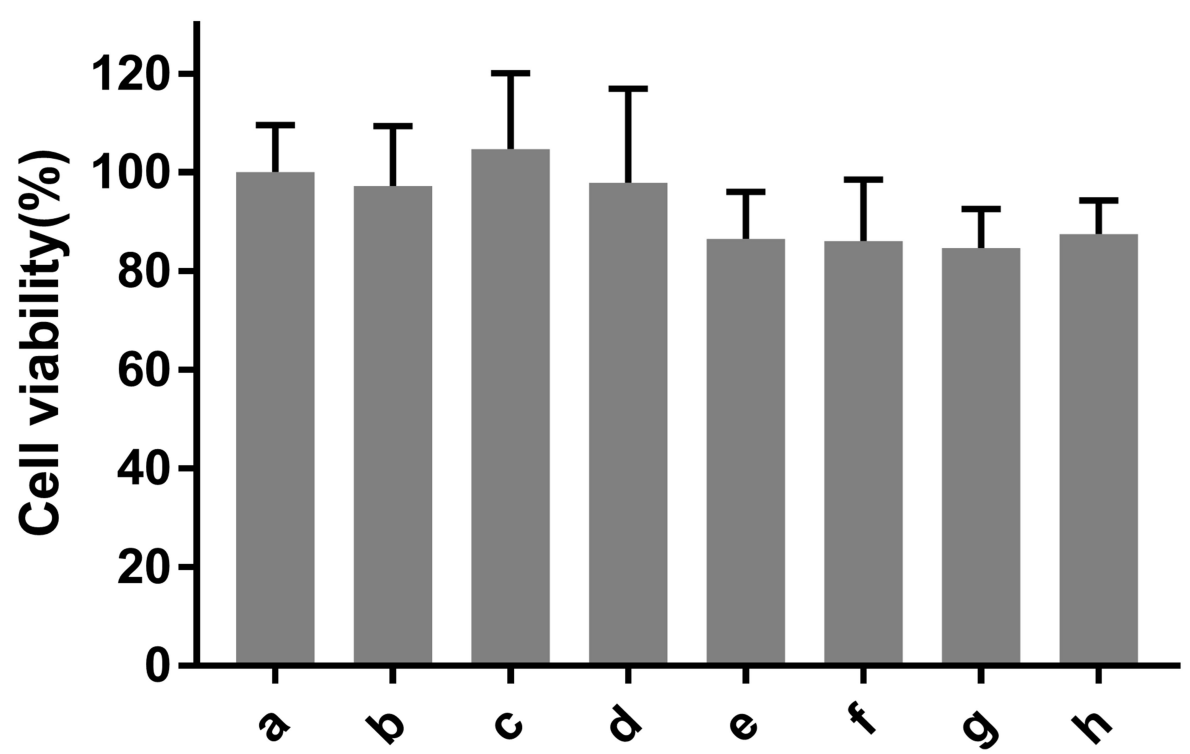

Figure 2 (AI) Temperature elevation of water and PDA-NPs at different concentrations as a function of irradiation time; (A2) Temperature change ( $\Delta T$ ) over $600 \mathrm{~s}$ versus the concentration of PDA-NPs; (A3) The photothermal response of the PDA-NPs for five repeat irradiations; (A4) Confocal images of calcein AM (green, live cells) and PI (red, dead cells) co-stained 4TI cells treated with PDA-NPs after laser irradiation. (B) Cell survival rate of PDA-NPs. Concentrations of PDA from a-h are I5, 30, 60, I20, 480 , and $960 \mu \mathrm{g} / \mathrm{mL}$, respectively. 
or DSPE-PEG-FA did not alter the EPI EE\%. The size and PDI of PDA-NPs and E/PCF-NPs remained unchanged and showed no obvious aggregation after one week at $4{ }^{\circ} \mathrm{C}$ (Supplementary Table S3), which indicated their excellent dispersibility under aqueous conditions. This is attributed to the PDAC cores being separated by PEGylated phospholipids, resulting in a combination of steric hindrance and molecular flexibility, which prevents the aggregation of the particles. ${ }^{57}$ As for the colloidal stability in FBSsupplemented RPMI of NPs (Supplementary Table S4), E/ PCF-NPs were maintained with a hydrodynamic size of $\sim 200 \mathrm{~nm}$, which was slightly larger than that observed in water, and increased slightly after $24 \mathrm{~h}$ of incubation.

\section{In vitro Release}

The release of EPI from E/PCF-NPs is shown in Figure 4A. The cumulative release from E/PCF-NPs was enhanced at $\mathrm{pH}$ 5.0 when $14.9 \%$ was released in $72 \mathrm{~h} ; 33.9$ and 5.6 times more than at $\mathrm{pH} 7.4$ and 6.5, respectively. Over the first $24 \mathrm{~h}$, a sustained release of EPI was observed without burst release at $\mathrm{pH} 5.0$, and EPI was hardly detected at $\mathrm{pH} 6.5$ or 7.0. With laser irradiation, the release of EPI from E/PCF-NPs increased by $29.3 \%$ in $24 \mathrm{~h}$ and $50.3 \%$ in $72 \mathrm{~h}$ at $\mathrm{pH} 5.0$, when compared to the E/PCF-NPs-treated group without NIR laser, respectively. The same trend was observed at $\mathrm{pH} 6.5$ and 7.4. The released EPI\% from E/PCF-NPs was increased 1.9- and 6.9-fold at $72 \mathrm{~h}$ with NIR light at $\mathrm{pH} 6.5$ and 7.4, compared to those of groups without NIR light, respectively. The release of EPI from E/PCF-NPs in absence of NIR irradiation exhibited a $\mathrm{pH}$-dependent release profile. With the $\mathrm{pH}$ of the release media decreased, the EPI release rate was increased, which resulted from the higher solubility of EPI as a weak base in the more acidic aqueous solution. ${ }^{58}$ This observation also indicated that the release could be controlled by laser irradiation. The enhanced EPI release rate could be a result of increased membrane fluidity caused by the high temperature response of PDAC to irradiation, ${ }^{59}$ with the consequent depolymerization facilitating the release of EPI.

\section{Cell Viability}

The effect of EPI from E/PCF-NPs on 4T1 cells was investigated using a standard MTT assay without exposure

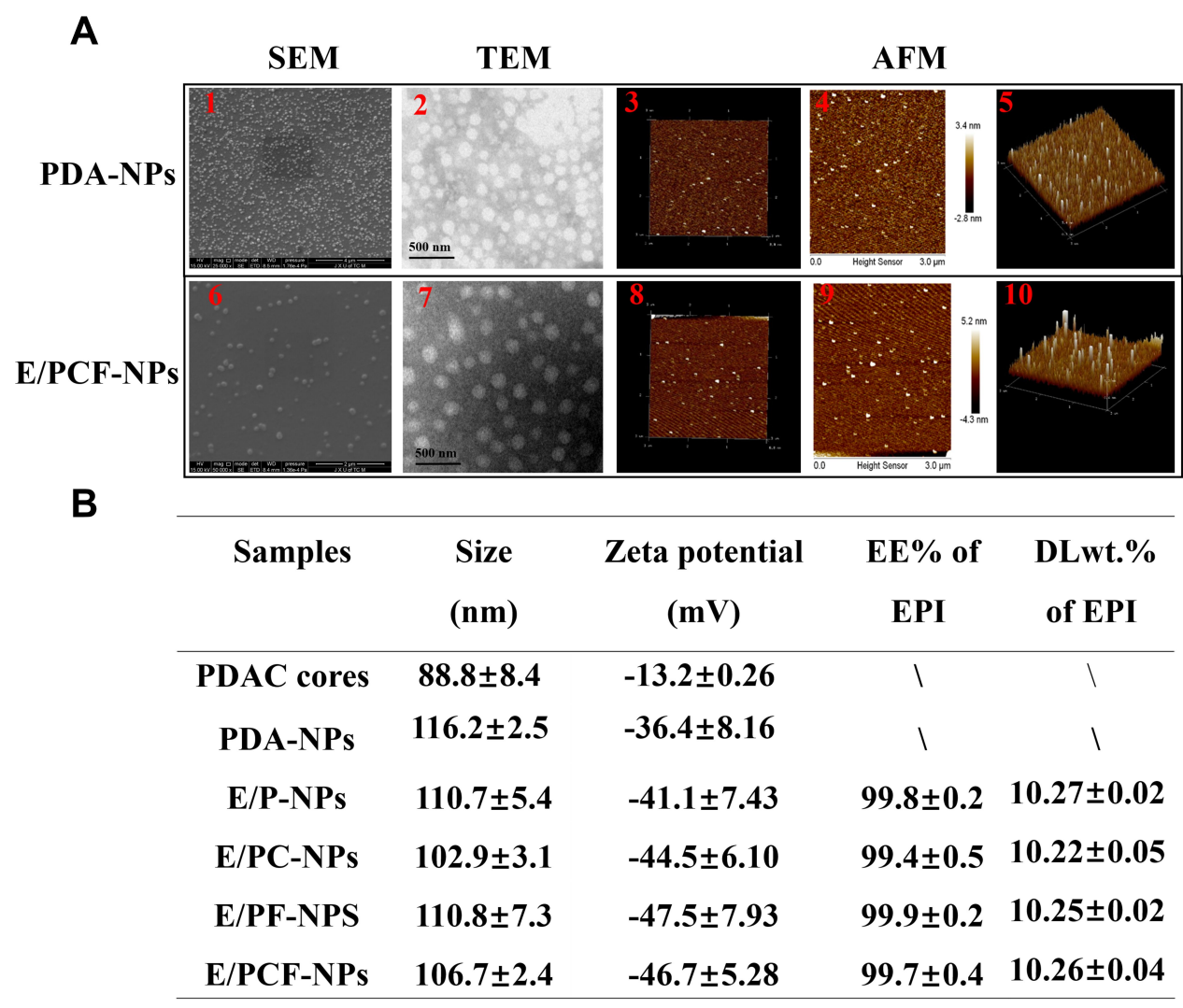

Figure 3 (AI) SEM image, (A2) TEM image, (A3) three-dimensional AFM topography image, (A4) AFM height image, and (A5) AFM three-dimensional height profile image of PDA-NPs. (A6) SEM image, (A7) TEM image, (A8) three-dimensional AFM topography image, (A9) AFM height image, and (A I0) AFM three-dimensional height profile image of E/PCF-NPs. (B) Characterization of PDAC cores and EPI-containing preparations. 
to the NIR laser. As shown in Figure 4B, the toxicity of E/ PCF-NPs was not significantly different to that of EPI alone $(p>0.05)$, indicating that modification of nanopreparation did not change the cytotoxicity of EPI.

The results in Figure 4C showed that PDA NPs (PDA: $200 \mu \mathrm{g} / \mathrm{mL}$ ) could significantly decrease cell viability by $72.2 \%$ after $3 \mathrm{~min}$ of laser irradiation at $808 \mathrm{~nm} 2.15 \mathrm{~W} /$ $\mathrm{cm}^{2}$. With the combination of chemo toxic agent EPI, the cell viability of E/PCF-NPs-treated group (PDA: $200 \mu \mathrm{g}$ / mL, EPI: $25 \mu \mathrm{g} / \mathrm{mL}$ ) was further decreased by $86.7 \%$ under NIR irradiation, compared to that of E/PCF-NPs-treated group without NIR irradiation. There was no significant difference in cell activity between the Free EPI group with or without NIR irradiation, which indicated that the cell viability could not be reduced by NIR irradiation without the existence of photothermal materials. The same result come from the cells treated with Free EPI at a higher concentration. The synergistic cytotoxicity for E/PCF-NPs results from photothermal conversion efficiency of PDAC cores and the chemo toxic ability of EPI. Furthermore, the modification of cRGD and FA facilitated the cellular uptake of E/PCF-NPs, when compared to that of unmodified nanoparticles (Figure 5), which also lead to the improved cytotoxicity.

\section{Detection of ROS}

Treatment with E/PCF-NPs under NIR laser irradiation led to significantly increased cell death than with E/PCF-NPs alone or EPI solution at a concentration of $25 \mu \mathrm{g} / \mathrm{mL}$ (Figure 4C). The elevated cell temperature in response to NIR irradiation leads to local hyperthermia or ROS generation that cause irreversible cancer cell death. ${ }^{60,61}$ The intracellular NPs cause improved EPI release under NIR laser irradiation (Figure 4A), which leads to greater toxicity to cells. In our study, we observed that the combination of NIR laser irradiation and E/PCF-NPs showed a more marked effect on tumor cells than chemotherapy or hyperthermia treatment alone. The results affirmed that the application of E/PCF-NPs along with NIR irradiation significantly inhibited the survival rate of cells. This result was consistent with the live/dead assay shown in Figure 2A4, in which treatment with E/PCF-NP along

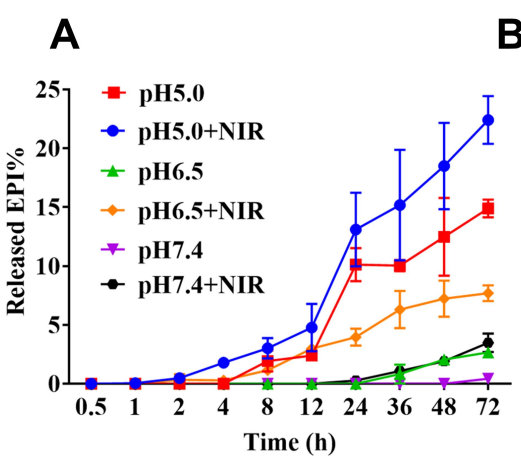

D

\begin{tabular}{|c|c|}
\hline Samples & $\begin{array}{c}\text { IC50 } \\
(\mu \mathrm{g} / \mathrm{mL})\end{array}$ \\
\hline Free EPI & $1.2 \pm 0.3$ \\
\hline E/PC-NPs & $1.0 \pm 0.2$ \\
\hline E/PF-NPs & $0.9 \pm 0.3$ \\
\hline E/PCF-NPs & $1.3 \pm 0.2$ \\
\hline
\end{tabular}

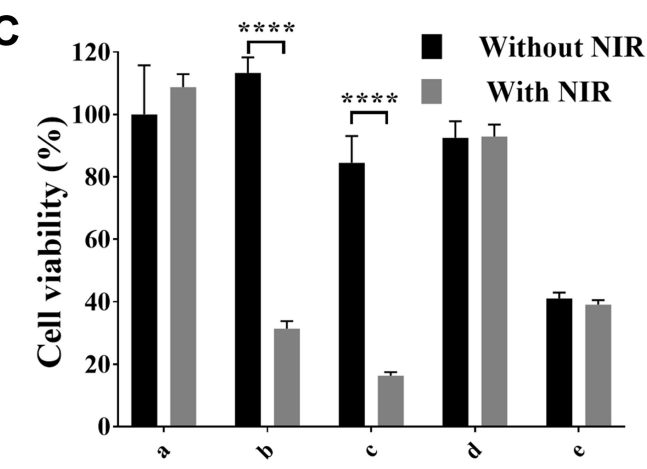

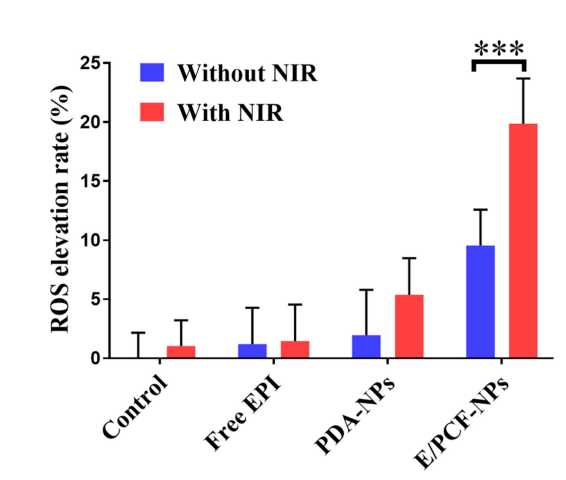

E

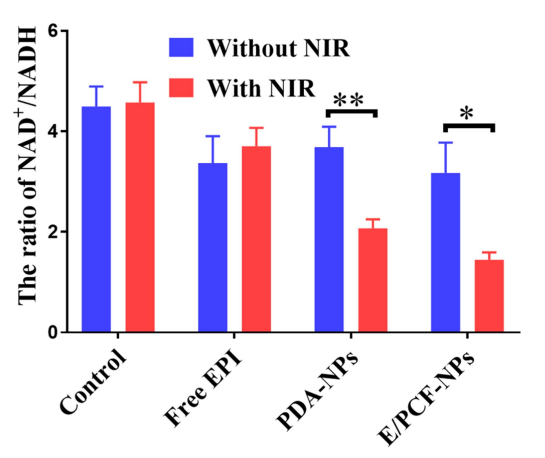

Figure 4 (A) The kinetics of cumulative release of EPI from E/PCF-NPs under different pH conditions (pH 7.4, 6.0, and 5.0) with or without NIR laser; (B) IC50 values for Free EPI, E/PC-NPs, E/PF-NPs, and E/PCF-NPs; (C) cell viability after PTT, a. control, b. PDA-NPs, c. E/PCF-NPs, d. EPI $25 \mu g / \mathrm{mL}$, e. EPI $200 \mu g / \mathrm{mL}$. *****p < 0.000 I; (D) ROS generation was monitored using the mean DCF fluorescence when $4 \mathrm{TI}$ cells were incubated with different preparations for I h in dark, followed by photo irradiation. $* * * p<$ 0.0005 . (E) The ratio of $\mathrm{NAD}^{+} / \mathrm{NADH}$ was monitored for $4 \mathrm{TI}$ cells incubated with different preparations for $24 \mathrm{~h}$, followed by photo irradiation treatment. ** $\mathrm{p}<0.005$, $*_{p}<0.05$. 
with NIR irradiation led to a much higher proportion of red cells than of green cells.

Intracellular ROS generation by E/PCF-NPs under NIR irradiation was determined by DCFH-DA. Figure 4D shows that compared to the control group, the ROS content of the EPI solution group with and without NIR laser irradiation showed no significant increase, while the ROS content of the PDA-NPs and E/PCF-NPs groups increased to $5.38 \%$ and $19.87 \%$ after excitation, respectively. This could be attributed to the decrease in cell viability caused by photothermal treatment promoting the increase of intracellular ROS. ${ }^{62}$

\section{Detection of $\mathrm{NAD}^{+} / \mathrm{NADH}$}

Oxidative stress induced by excessive ROS can cause mitochondrial dysfunction. ${ }^{63}$ Therefore, we analyzed the $\mathrm{NAD}^{+} / \mathrm{NADH}$ content of the cells. The same trend was found in $\mathrm{NAD}^{+} / \mathrm{NADH}$ detection (Figure 4E); compared with the control group, the $\mathrm{NAD}^{+} / \mathrm{NADH}$ content of the EPI solution, PDA-NPs, and E/PCF-NPs groups were slightly reduced by $18-28 \%$, while there were no significant differences between the EPI solution and control groups under NIR irradiation. However, the $\mathrm{NAD}^{+} /$ NADH concentrations of cells treated with PDA-NPs and E/PCF-NPs were significantly reduced to $55.7 \%$ and $38.9 \%$ of that of cells treated with Free EPI, respectively. Reduced NADH is abundant in mitochondria and acts as a strong reductant in the biosynthesis of ATP. The NADH consumed under NIR laser irradiation disrupts the redox homeostasis and enhances oxidation stress-related apoptosis. $^{64,65}$

\section{Cell Apoptosis}

Apoptosis, or programmed cell death, is a complex process regulated by multiple genes. We used the annexin V/PI double staining kit to quantify 4T1 cell apoptosis in response to treatment with E/PCF-NPs and NIR laser irradiation. The stained cells were divided into four subgroups; the viable group, the early apoptotic group, the late apoptotic/necrotic group, and the dead cells/debris group, which were localized in the lower left, lower right, upper right, and upper left quadrants, respectively. ${ }^{32}$ The percentages of specific cell populations at various stages of apoptosis are shown in Figure 6 (Detailed data of other groups can be found in Figure S1). Compared with the control group, the cells without treatment were mostly alive $(98.2 \%$ of viable cells) under irradiation. However, after treatment with a PDA-containing preparation and NIR laser irradiation, the percentage of viable cells decreased, and that of the apoptotic cells increased significantly. The apoptosis rate in the control group was $0.2 \pm 0.1 \%$. E/PCF-NPs treatment increased the rate of apoptosis from $24.1 \%$ to $73.2 \%$ after irradiation, which was 2.3 times higher than that for the combination of PDA-NPs and NIR; however, there was no difference when EPI without or with NIR was compared with the control group ( $p>0.05$, Figure S1). The results showed that E/PCF-NPs induced apoptosis and led to a significant increase in the late-stage apoptosis and necrosis under irradiation. These results supported the outcome of the MTT assays.

\section{Cellular Uptake}

The internalization of E/PCF-NPs was evaluated using EPI as a fluorescent probe in 4T1 cellular uptake using confocal laser scanning microscopy (CLSM) and flow cytometry. The red fluorescence signal of cells incubated with the same concentration of Free EPI was stronger than that of E/P-NPs after $4 \mathrm{~h}$ of incubation, which is assumed to be internalized by passive diffusion. However, the cellular uptake of Free EPI did not increase with time. E/P-NPs were endocytosed into cells in a time-dependent manner (Figure 5A and B). All the membrane-modified NPs showed increased cellular uptake (\%) compared to E/ P-NPs after $4 \mathrm{~h}$ of incubation, while E/PCF-NPs showed the highest uptake. Compared with E/PF-NPs, the fluorescence signals of E/PC-NPs were stronger within $4 \mathrm{~h}$. As demonstrated in Figure 5C, similar results were observed by flow cytometry. It was confirmed that modified NPs, subjected to either single or dual modification, exhibited greater uptake of EPI compare to Free EPI and E/P-NPs. Cells treated with E/PC-NPs showed stronger fluorescence than those treated with E/PF-NPs. The cRGD targeting group enhanced the cellular uptake efficiency of the NPs through receptor-mediated endocytosis and transportation through membranes. ${ }^{66}$ FA could selectively bind to the glycosylphosphatidylinositol-linked folate receptor on the cells surfaces but not facilitate tumor penetration. ${ }^{67}$ When the incubation time was prolonged to $12 \mathrm{~h}$, there was no significant difference among uptake of E/PC-NPs, E/PFNPs, and E/PCF-NPs. This indicates that single or dual modification of NPs would lead to similar cellular uptake if the incubation time is long enough. Negligible fluorescence was observed in cells after incubating with PBS. Almost no autofluorescence was observed. Collectively, these results indicate that the E/PCF-NPs could be internalized by the cancer cells more rapidly and effectively 
A

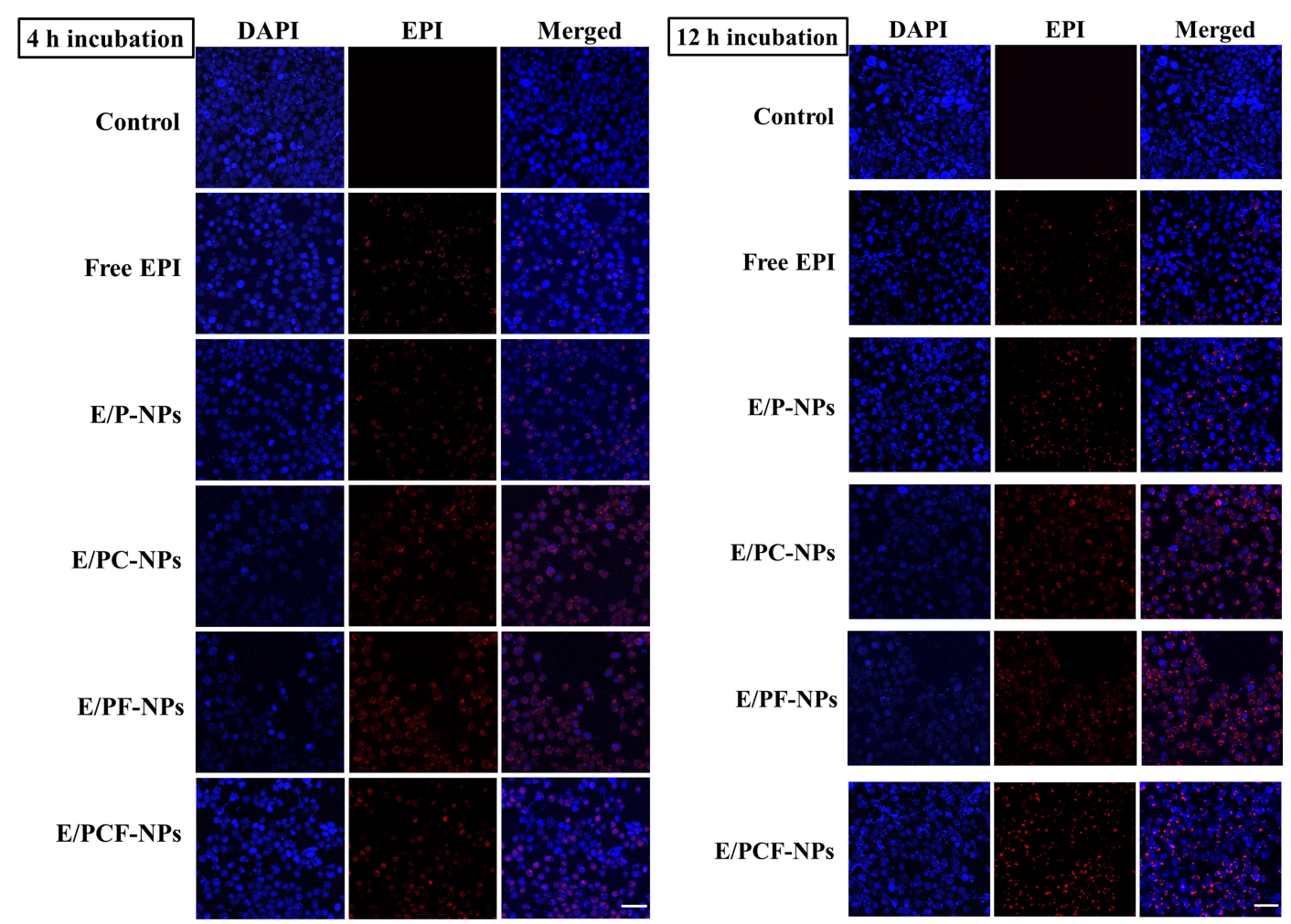

B

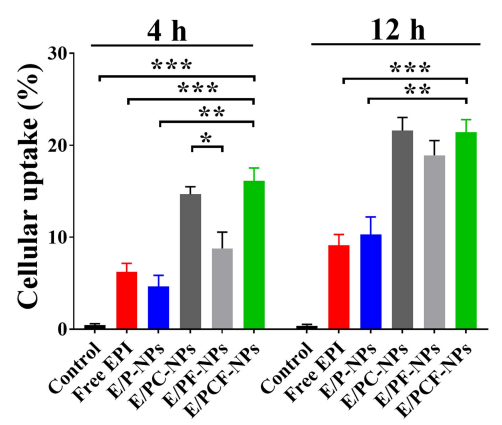

C

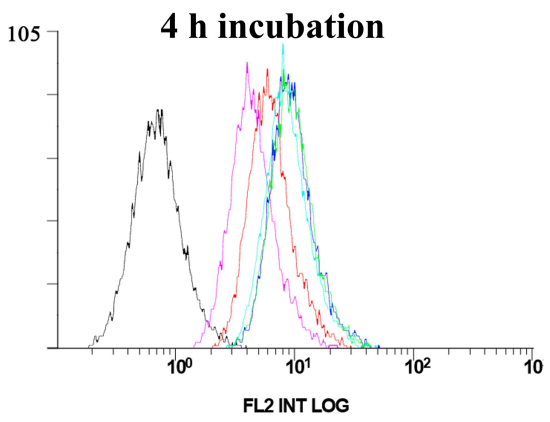

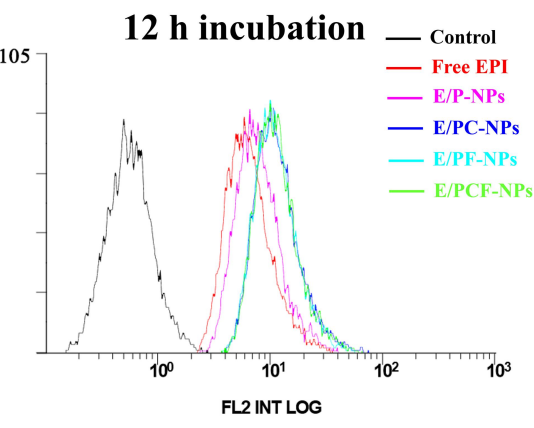

Figure 5 (A) In vitro CLSM images of $4 \mathrm{TI}$ cells treated with EPI containing preparations for 4 and $12 \mathrm{~h}$ at $37^{\circ} \mathrm{C}, 5 \% \mathrm{CO}_{2}$. All images show the nuclei (blue), EPI (red), and merged images. Scale bar; $50 \mu \mathrm{m}$; (B) quantitative results for the cellular uptake of EPI-loaded preparations expressed as a percentage of total cell number, $* * * p<0.0005$, ${ }^{* *} p<0.005,{ }^{*} p<0.05$; (C) flow cytometry analysis of cellular uptake of EPI containing preparations in $4 \mathrm{TI}$ cells.

through endocytosis than Free EPI, E/PC-NPs, and E/PFNPs. Targeting and elevated cellular uptake resulting from DSPE-PEG2000-FA/cRGD modification was confirmed.

\section{Pharmacokinetics Study}

To determine the contribution of the long-circulation ability of E/PCF-NPs, a pharmacokinetic study was carried out. Plasma was collected at scheduled time points and drug concentrations were quantified by UHPLC/MS. The nanopreparations showed significantly prolonged $\mathrm{MRT}_{0 \rightarrow \mathrm{t}}$ in the bloodstream and a much higher $\mathrm{C}_{\max }$ compared to the EPI solution $(p<0.05)$ (Figure 7A and Table 1). The long circulating properties exhibited by the NPs were attributed to DSPE-PEG-cRGD and DSPE-PEG-FA modification. The physical properties of these NPs may contribute to changes in the circulation lifetimes of EPI or EPI NPs. There were no clear differences in EE\% or size distribution for any of the NPs used in this study. However, analysis of the zeta potential of unmodified and modified NPs containing DSPE-PEG showed slight, but significant, differences. The $\mathrm{AUC}_{0 \rightarrow \mathrm{t}}$ significantly increased for modified EPI nanopreparations that contained DSPE-PEG. PEG coatings were found to be effective in shielding the NPs from the RES via steric repulsion. PEG created a hydrated outer shell, which 

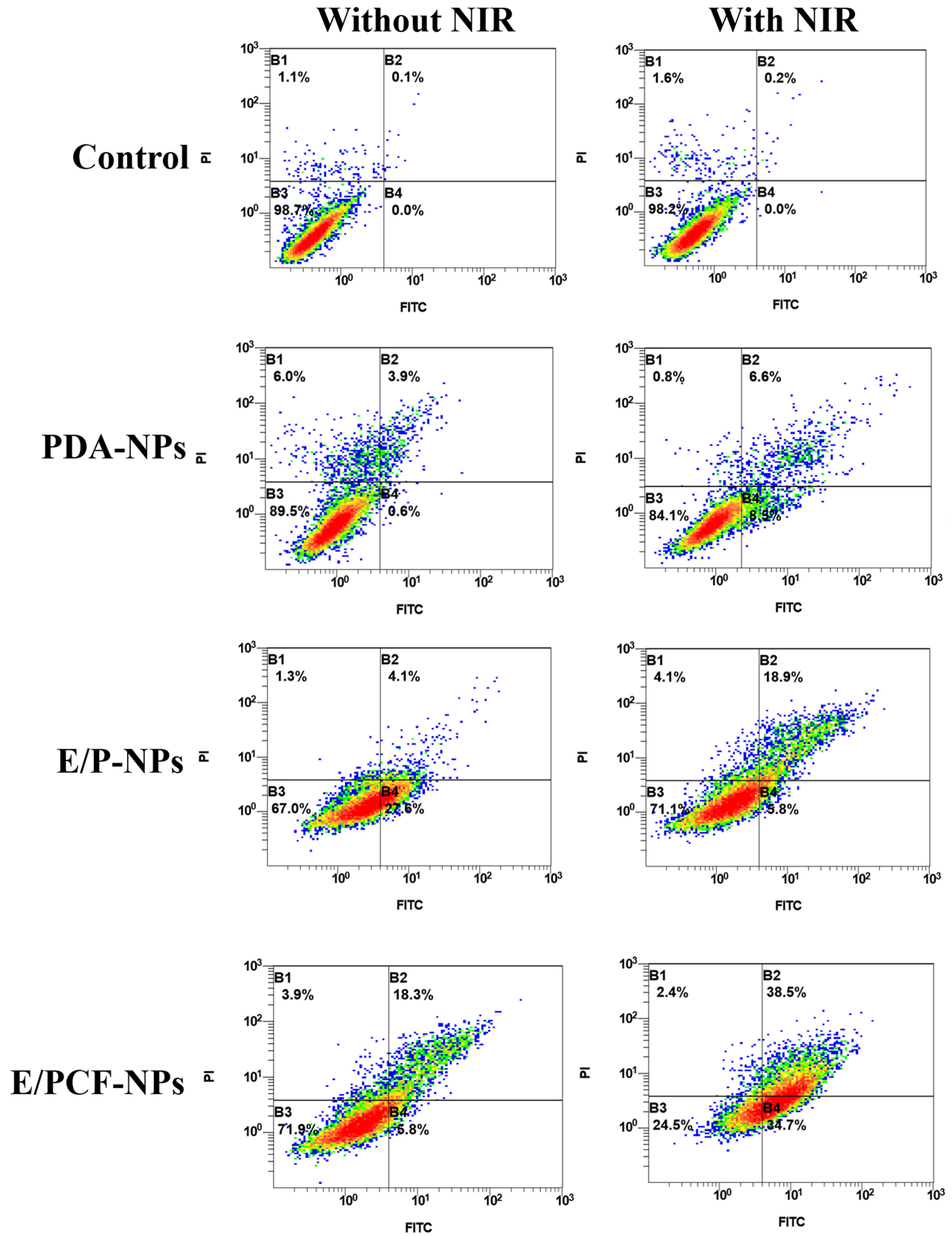

Figure 6 Apoptosis of 4TI cells treated with different preparations by annexin V/PI staining using flow cytometry. 
also shielded the NPs from being recognized and cleared by the RES. All the effects of PEG resulted in an extended drug half-life and improved tissue distribution. ${ }^{68,69}$ Due to the modification of PEG, the $\mathrm{AUC}_{0 \rightarrow \mathrm{t}}$ and $\mathrm{C}_{\max }$ of E/P-NPs, E/ PC-NPs, E/PF-NPs, and E/PCF-NPs were significantly higher than those of Free EPI. However, although the $\mathrm{C}_{\max }$ of E/PCF-NPs was seven-fold higher than that of EPI solution, it was lower than that of E/P-NPs, E/PCNPs, and E/PF-NPs by $82.7 \%, 37.2 \%$, and $40.7 \%$, respectively. The relatively lower $\mathrm{C}_{\max }$ of E/PCF-NPs is believed to be the result of the faster distribution of EPI from the bloodstream to tumor tissues. As observed in the tissue distribution study, the distribution of E/PCF-NPs in tumors was much higher than those of E/P-NPs, E/PC-NPs, and E/ PF-NPs (Figure 7B). Due to the targeting modification, the double-modified NPs, E/PCF-NPs, exhibited higher tumor accumulation and stronger targeting ability than those of single-modified NPs, E/PC-NPs, and E/PF-NPs. Therefore, the modified NPs showed lower blood concentrations, especially for the double-modified NPs. Compared with that of EPI in E/P-NPs, the $\mathrm{V}_{\mathrm{z}}$ of EPI in E/PCF-NPs decreased significantly by $22.4 \%$. The CL of E/PCF-NPs was 9.4-fold slower than that of free drug. These results suggest that the double-modified nanocarriers, E/PCF-NPs, showed prolonged circulation time in blood compared to free drug, but faster drug distribution than the unmodified NPs, E/ P-NPs.

\section{Tissue Distribution}

A tissue distribution study was carried out to determine the $\mathrm{E} /$ PCF-NPs targeting ability. Due to the polyethylene glycol membrane modification, E/PCF-NPs are expected to exhibit prolonged systemic circulation and effective delivery of the cargo to the tumor due to the presence of the targeting ligands cRGD and FA in combination with the EPR effect. ${ }^{70}$ EPI concentrations were detected in tumors and major organs (Figure 7B). EPI from E/PCF-NPs mainly accumulated in the tumors within $0.5 \mathrm{~h}$, which is 3.6-, 10.9-, and 28.7-fold greater than EPI from E/PF-NPs, E/PC-NPs, and E/P-NPs, respectively. Due to the large blood volume and fast blood flow of the liver, ${ }^{71} 31.7$-fold more NPs distributed in the liver compared to the tumor for E/P-NPs. Compared with E/ P-NPs, E/PC-NPs, and E/PF-NPs; EPI from E/PCF-NPs showed better tumor-targeting at early time points, which was consistent with the lower $\mathrm{C}_{\max }$ of EPI from E/PCF-NPs in the pharmacokinetic study. At later time points, with the help of the PEGylated and targeting ligands, most of the NPs still accumulated in the tumor, though a certain number of NPs accumulated in the liver or spleen. The tumor EPI concentration for E/PCF-NPs was at least 1.8-fold greater

A

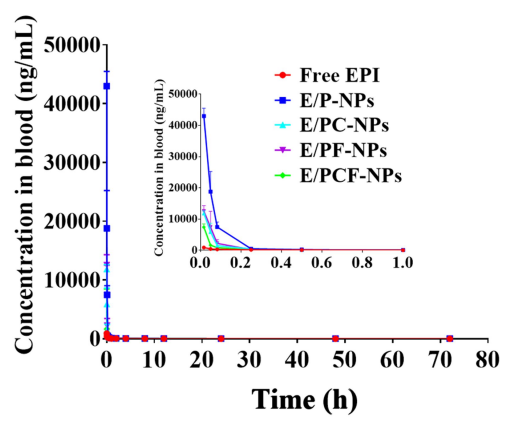

B
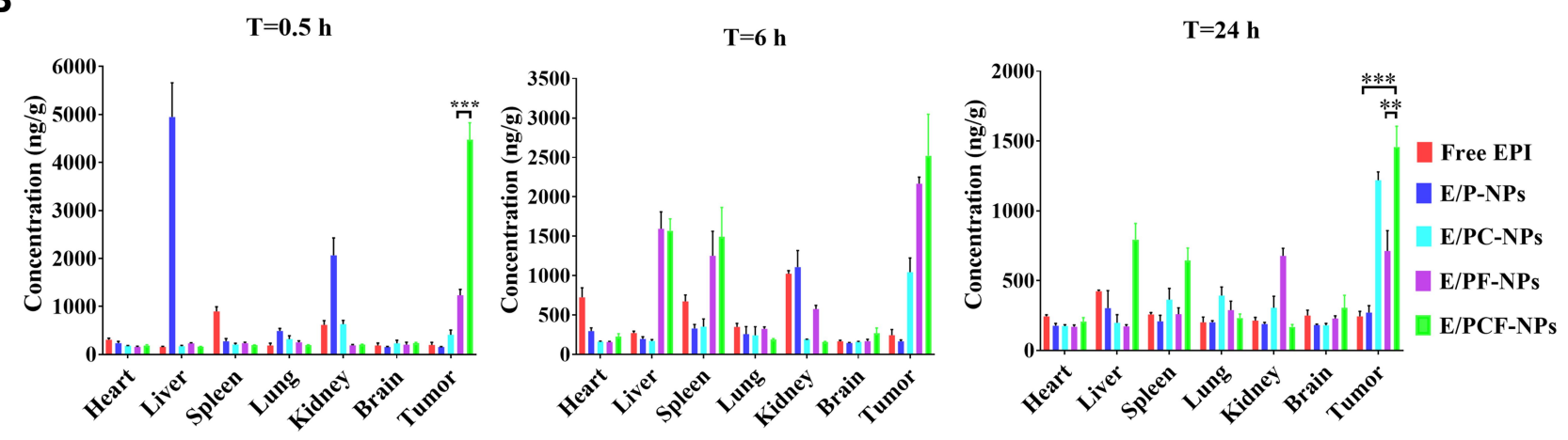

Figure 7 (A) Blood concentration-time profiles of formulations of EPI after intravenous injection at $10 \mathrm{mg} / \mathrm{kg}$ in rats ( $\mathrm{n}=3$ ). (B) Biodistribution of EPI from different preparations in tumors and major organs detected by UHPLC/MS at different time points after intravenous injection $(\mathrm{n}=3), * * p<0.005, * * * p<0.0005$. 
Table I Pharmacokinetic Parameters of EPI-Containing Preparations (Mean \pm SD)

\begin{tabular}{|l|l|l|l|l|l|}
\hline & $\mathbf{C}_{\max }(\mathbf{n g} / \mathbf{m L})$ & AUC $_{\mathbf{0} \rightarrow \mathbf{t}}(\mathbf{n g} / \mathbf{m L} \mathbf{h})$ & $\mathbf{M R T}_{\mathbf{0} \rightarrow \mathbf{t}}(\mathbf{h})$ & $\mathbf{V}_{\mathbf{z}} \mathbf{( L )}$ & $\left.\mathbf{C L}_{\mathbf{( L}} \mathbf{h}\right)$ \\
\hline Free EPI & $925.9 \pm 169.1$ & $750.5 \pm 192.4$ & $40.5 \pm 17.3$ & $110 \pm 40.3$ & $10.16 \pm 1.6$ \\
E/P-NPs & $43,000.3 \pm 2040.6 \# \delta$ & $5199.9 \pm 124.3 \#^{*}$ & $359.3 \pm 192.8 \# *$ & $254 \pm 50.1$ & $0.42 \pm 0.3 \#$ \\
E/PC-NPs & $11,844.4 \pm 652.4 \#^{*}$ & $3804 \pm 89.4 \#$ & $101.0 \pm 49.6 \#$ & $198 \pm 40.1$ & $0.97 \pm 0.3 \#$ \\
E/PF-NPs & $12,554.3 \pm 1428.6 \# *$ & $3771.3 \pm 356.9 \#$ & $133.5 \pm 18.1 \#$ & $187 \pm 10.0$ & $1.21 \pm 0.1 \#$ \\
E/PCF-NPs & $7443.6 \pm 840.3 \#$ & $3367.5 \pm 244.1 \#$ & $154.5 \pm 6.2 \#$ & $197 \pm 8.9$ & $1.08 \pm 0.1 \#$ \\
\hline
\end{tabular}

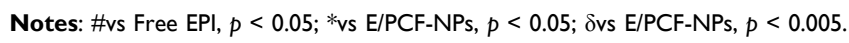

than at other major organs. Furthermore, non-tumor selective distribution was identified for Free EPI, with higher EPI accumulation in the heart, liver, and spleen at all time points. Based on the pharmacokinetic and biodistribution profiles of E/PCF-NPs, we conclude that prolonged blood circulation and increased tumor accumulation are the major benefits of using an E/PCF-NPs delivery system.

\section{In vivo Anti-Tumor Effects}

The anti-tumor effect of dual ligand functionalization of E/ PCF-NPs was characterized in vivo using a 4T1 orthotopic tumor model. For the tumors at the early stage $\left(150 \mathrm{~mm}^{3}\right)$, PDA-NPs, E/P-NPs, E/PC-NPs, E/PF-NPs, and E/PCF-NPs were intravenously injected in tumor-bearing mice three times at seven-day intervals at an EPI dose of $5 \mathrm{mg} / \mathrm{kg}$ with or without NIR laser irradiation. Changes in tumor volume, tumor weight, and body weight were monitored for each group. As shown in Figure 8A-C, mice experienced rapid tumor growth in the control group with or without NIR, suggesting that only the use of NIR laser irradiation without PDA exhibits no effect on the growth of tumors. Similarly, treatment with PDA-NPs alone without NIR had no influence on attenuating tumor growth. In contrast, group subjected to the PDA-NPs treatment along with irradiation showed a significant delay in tumor growth, with a $55.2 \%$ decrease in tumor weight, compared with single PDA-NPs groups.
A

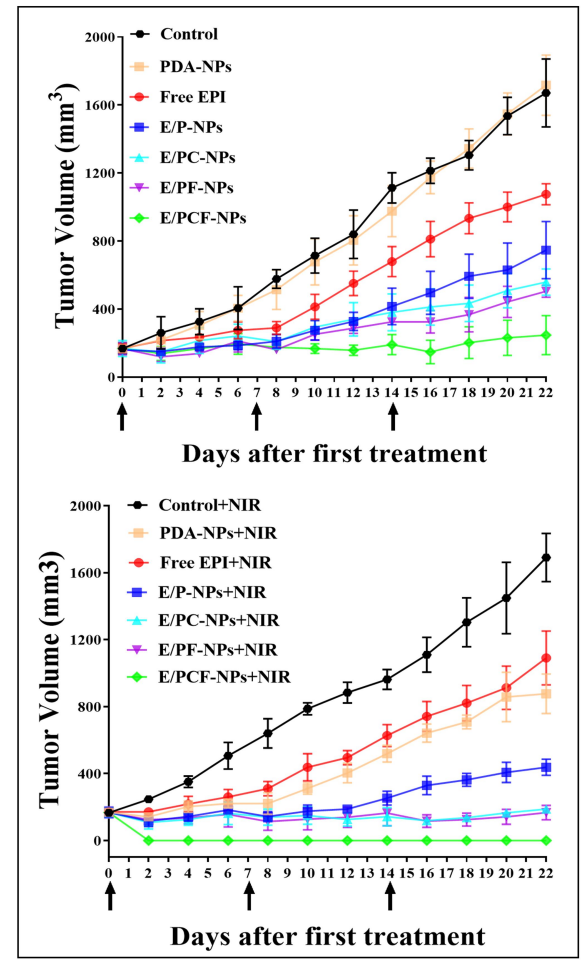

B

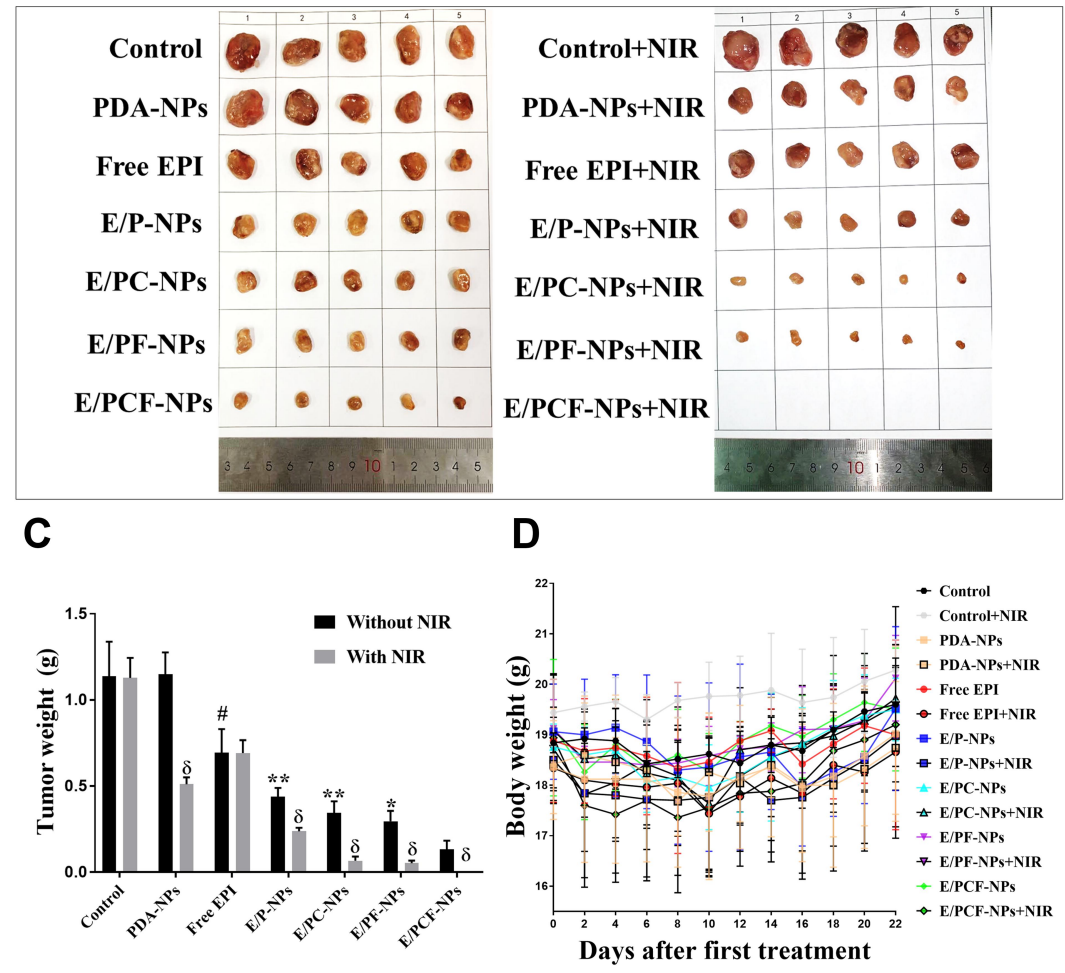

Figure 8 (A) Inhibition of early stage tumor growth in different groups with EPI at $5 \mathrm{mg} / \mathrm{kg}(\mathrm{n}=4)$. Arrows indicate the days of injection. (B) Tumors were collected and photographed and (C) weighed; \#vs Control group, $p<0.05$; *vs E/PCF-NPs, $p<0.005$; **vs E/PCF-NPs, $p<0.000$ I; $\delta$ vs the same treatment without NIR, $p<0.005$. (D) Body weight changes during treatment for each group. 


\section{A}
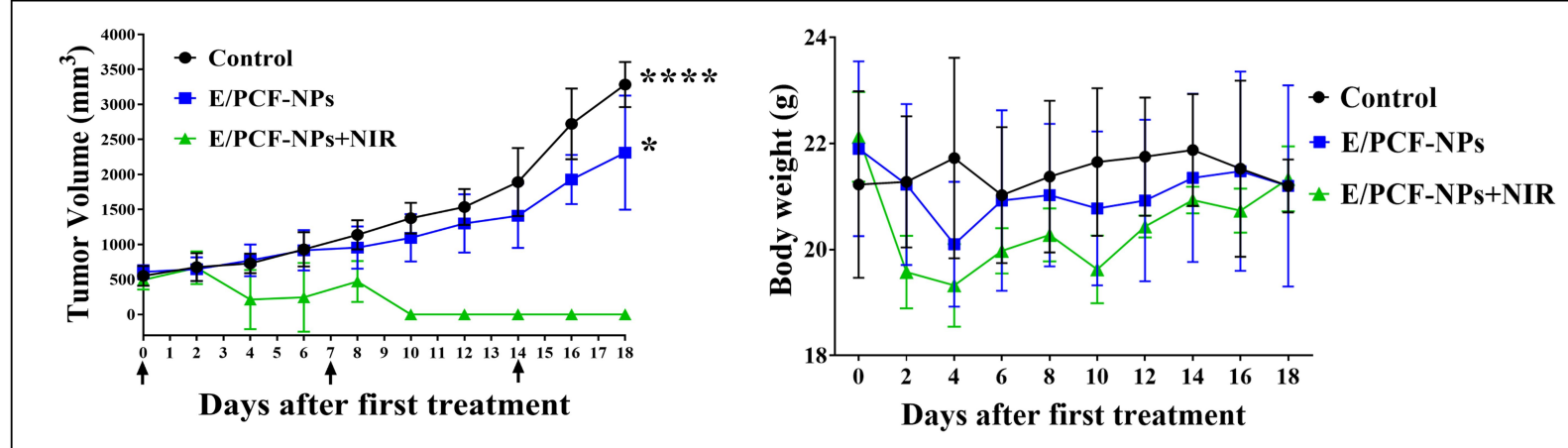

B
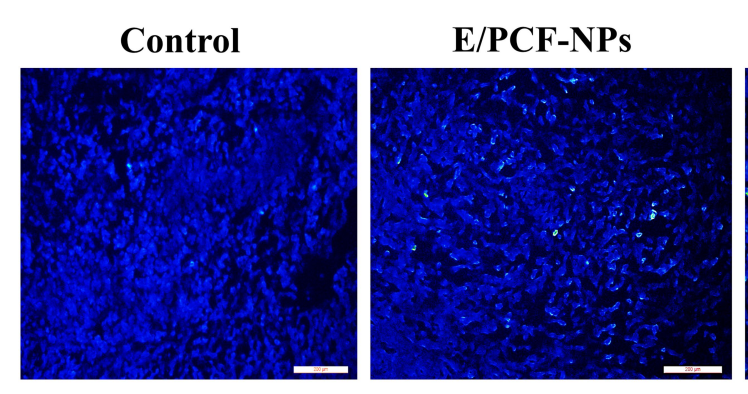

E/PCF-NPs+NIR

Figure 9 (A) Inhibition of late-stage tumor growth in different groups with EPI at $5 \mathrm{mg} / \mathrm{kg}(\mathrm{n}=4)$. Arrows indicate the days of injection. *vs E/PCF-NPs $+\mathrm{NIR}$, $p<0.05$; $* * * *$ vs E/PCF-NPs+NIR, $p<0.0001$. (B) TUNEL-positive cells $(\%)$ in tumors of each group $(\mathrm{n}=4)$. **** $p<0.0001, * * * p<0.0005,{ }^{*} p<0.05$.

The EPI-loaded NPs, E/P-NPs, E/PC-NPs, E/PF-NPs, and E/ PCF-NPs, without NIR, showed better anti-tumor effects than Free EPI, which is believed to result from the EPR effect and active targeting to tumors. E/PCF-NPs exhibited a significantly enhanced therapeutic efficiency, with a $61.7 \%$ and $55.3 \%$ decrease in tumor weight compared to E/PC-NPs and E/PF-NPs, respectively $(p<0.5)$. Furthermore, the tumors in the E/PCF-NPs group with NIR were almost destroyed with only scar tissue remaining after two weeks of treatment. The results demonstrate the enhanced effect of combined photothermal and chemotherapeutic treatment with E/PCF-NPs and NIR laser irradiation, compared with any single modality treatment. Since high toxicity generally leads to weight loss, we also measured the body weights of the mice for all groups during treatments. There was $4.9 \%$ and $8.3 \%$ weight loss for Free EPI and E/PCF-NPs, respectively, under NIR laser irradiation within the first week of treatment. As treatment progressed, no further weight loss was observed (Figure 8D), implying that the toxicity or side effects of these NPs were not serious. This was also consistent with the results obtained from the H\&E staining of major organs (data not shown). H\&E staining of the control, PDANPs, E/P-NPs, E/PC-NPs, E/PF-NPs, and E/PCF-NPs groups with or without NIR showed no obvious kidney injury, pulmonary toxicity, cardiac damage, or inflammatory infiltrates in the spleen.
To further study the anti-tumor efficacy of E/PCF-NPs under NIR laser irradiation, we evaluated the treatment of the late stage of tumors $\left(500 \mathrm{~mm}^{3}\right)$. The mice were divided into three groups: a control group and E/PCF-NPs groups with and without NIR laser irradiation. The design of the treatment was the same as previously described. E/PCF-NPs slightly inhibited the growth of tumors when compared with the control group; however, E/PCF-NPs combined with photothermal treatment almost destroyed the tumor with only scar tissue remaining after two weeks of treatment (Figure 9A). This showed that for late-stage tumors, E/PCF-NPs under NIR irradiation exhibited a greater impact on tumor progression than NPs alone. There was no obvious weight loss observed at the end of treatment. The mice in E/PCF-NPs under the NIR laser group also experienced some degree of weight loss in the early treatment within one week, which might be due to the shrinking of tumors in mice. The TUNEL assay (Figure 9B) revealed that E/PCF-NPs under NIR laser irradiation exhibited the most effective cytotoxicity and induced 38 and 8.7 times more apoptosis in cells compared with the control group and E/PCF-NPs without NIR group, respectively. This finding is consistent with that obtained from the cellular experiments and is explained by the fact that ROS derived from NIR laser treatment can damage the tumor tissues, and the heat released by the photothermal pathway also causes 
cell significant damage when it reaches a threshold temperature of $\sim 42^{\circ} \mathrm{C}$ for a prolonged period. ${ }^{26}$

\section{Conclusions}

A chemo and photothermal therapeutic agent based on EPI-loaded PDA NPsthat was double modified with FA and cRGD was successfully synthesized. The E/PCF-NPs exhibited excellent biocompatibility and low toxicity, as well as high ROS generation and high photo-thermal conversion efficiencies. The antitumor efficiency of the E/ PCF-NPs upon NIR laser irradiation was carefully evaluated both in vitro and in vivo, and the results showed that complete tumor regression for tumor-bearing mice could be realized, making the combined treatment more effective than single-modified NPs, or chemo or photothermal treatment alone. Therefore, our prepared E/PCF-NPs are promising therapeutic agents for future cancer therapy.

\section{Funding}

This research was supported by the National Natural Science Foundation of China (No. 81603054 and 81760639), the Jiangxi Provincial Department of Science and Technology (20171BAB215066, 20202ACBL216015, and 20202BA BL206157), the Jiangxi Provincial Department of Education (GJJ190666 and GJJ190663), Research project of traditional Chinese medicine of Jiangxi health and Family Planning Commission (2016A008), Young Jinggang Scholar of Jiangxi Province and "1050" Talents Program (Jing Zhang and Xiang Li), New Century Talents Project of Jiangxi Province (Xiang $\mathrm{Li}$ and Jing Zhang), and the Jiangxi University of Traditional Chinese Medicine (JXSYLXKZHYAO055, JXSYLXK-ZHYAO056, and JXSYLXKZHYAO019).

\section{Disclosure}

The authors declare no conflict of interest.

\section{References}

1. Gelmon K, Dent R, Mackey JR, Laing K, McLeod D, Verma S. Targeting triple-negative breast cancer: optimising therapeutic outcomes. Ann Oncol. 2012;23(9):2223-2234. doi:10.1093/annonc/ mds067

2. Criscitiello C, Azim HA, Schouten PC, Linn SC, Sotiriou C. Understanding the biology of triple-negative breast cancer. Ann Oncol. 2012;23:13-18. doi:10.1093/annonc/mds188

3. Brewster AM, Chavez-MacGregor M, Brown P. Epidemiology, biology, and treatment of triple-negative breast cancer in women of African ancestry. Lancet Oncol. 2014;15(13):E625-E634. doi:10. 1016/s1470-2045(14)70364-x
4. Hurvitz S, Mead M. Triple-negative breast cancer: advancements in characterization and treatment approach. Curr Opin Obstet Gynecol. 2016;28(1):59-69. doi:10.1097/gco.0000000000000239

5. Martin HL, Smith L, Tomlinson DC. Multidrug-resistant breast cancer: current perspectives. Breast Cancer Dove Med. 2014;6:1-13. doi: $10.2147 / \mathrm{bctt} . S 37638$

6. Su SS, Ding YP, Li YY, Wu Y, Nie GJ. Integration of photothermal therapy and synergistic chemotherapy by a porphyrin self-assembled micelle confers chemosensitivity in triple-negative breast cancer. Biomaterials. 2016;80:169-178. doi:10.1016/j.biomaterials.2015.11.058

7. Cheng W, Liang CY, Wang XS, et al. A drug-self-gated and tumor microenvironment-responsive mesoporous silica vehicle: "four-inone" versatile nanomedicine for targeted multidrug-resistant cancer therapy. Nanoscale. 2017;9(43):17063-17073. doi:10.1039/ c7nr05450e

8. Pan QB, Zhang J, Li X, et al. Construction of novel multifunctional luminescent nanoparticles based on DNA bridging and their inhibitory effect on tumor growth. RSC Adv. 2019;9(26):15042-15052. doi:10.1039/c9ra01381d

9. Zhang J, Li X, Huang L. Anticancer activities of phytoconstituents and their liposomal targeting strategies against tumor cells and the microenvironment. Adv Drug Deliv Rev. 2020. doi:10.1016/j. addr.2020.05.006

10. Kesharwani SS, Dachineni R, Bhat GJ, Tummala H. Hydrophobically modified inulin-based micelles: transport mechanisms and drug delivery applications for breast cancer. J Drug Deliv Sci Technol. 2019;54:101254-101262. doi:10.1016/j.jddst.2019.101254

11. Muley P, Kumar S, Kourati FE, Kesharwani SS, Tummala H. Hydrophobically modified inulin as an amphiphilic carbohydrate polymer for micellar delivery of paclitaxel for intravenous route. Int J Pharm. 2016;500(1-2):32-41. doi:10.1016/j.ijpharm.2016.01.005

12. Nieberler M, Reuning U, Reichart F, et al. Exploring the role of RGD-recognizing integrins in cancer. Cancers. 2017;9(12):33. doi:10.3390/cancers9090116

13. Brooks PC, Clark RAF, Cheresh DA. Requirement of vascular integrin alpha-v-beta-3 for angiogenesis. Science. 1994;264 (5158):569-571. doi:10.1126/science.7512751

14. Eliceiri BP, Cheresh DA. The role of alphav integrins during angiogenesis: insights into potential mechanisms of action and clinical development. J Clin Invest. 1999;103(9):1227-1230. doi:10.1172/ JCI6869

15. Zhang J, Zhang P, Zou Q, et al. Co-delivery of gemcitabine and paclitaxel in cRGD-modified long circulating nanoparticles with asymmetric lipid layers for breast cancer treatment. Molecules. 2018;23(11):19. doi:10.3390/molecules23112906

16. Norton N, Youssef B, Hillman DW, et al. Folate receptor alpha expression associates with improved disease-free survival in triple negative breast cancer patients. NPJ Breast Cancer. 2020;6(1):4. doi:10.1038/s41523-020-0147-1

17. Werner ME, Copp JA, Karve S, et al. Folate-targeted polymeric nanoparticle formulation of docetaxel is an effective molecularly targeted radiosensitizer with efficacy dependent on the timing of radiotherapy. ACS Nano. 2011;5(11):8990-8998. doi:10.1021/ nn203165z

18. Li X, Tian X, Zhang J, et al. In vitro and in vivo evaluation of folate receptor-targeting amphiphilic copolymer-modified liposomes loaded with docetaxel. Int J Nanomedicine. 2011;6:1167-1184. doi:10.2147/ ijn.S21445

19. Gao ZG, You CQ, Wu HS, Wang MX, Zhang XY, Sun BW. FA and cRGD dual modified lipid-polymer nanoparticles encapsulating polyaniline and cisplatin for highly effective chemo-photothermal combination therapy. J Biomater Sci Polym Ed. 2018;29(4):397-411. doi:10.1080/09205063.2017.1421348

20. Nagini S. Breast cancer: current molecular therapeutic targets and new players. Anticancer Agents Med Chem. 2017;17(2):152-163. doi:10.2174/1871520616666160502122724 
21. Khosravi-Shahi P, Cabezon-Gutierrez L, Custodio-Cabello S. Metastatic triple negative breast cancer: optimizing treatment options, new and emerging targeted therapies. Asia Pac J Clin Oncol. 2018;14 (1):32-39. doi:10.1111/ajco.12748

22. Zhang J, Li X, Huang L. Non-viral nanocarriers for siRNA delivery in breast cancer. J Control Release. 2014;190:440-450. doi:10.1016/ j.jconrel.2014.05.037

23. Denkova AG, de Kruijff RM, Serra-Crespo P. Nanocarrier-mediated photochemotherapy and photoradiotherapy. Adv Healthc Mater. 2018;7(8):21. doi:10.1002/adhm.201701211

24. Su SS, Tian YH, Li YY, et al. "Triple-punch" strategy for triple negative breast cancer therapy with minimized drug dosage and improved antitumor efficacy. ACS Nano. 2015;9(2):1367-1378. doi:10.1021/nn505729m

25. Sun W, Du Y, Liang XL, et al. Synergistic triple-combination therapy with hyaluronic acid-shelled PPy/CPT nanoparticles results in tumor regression and prevents tumor recurrence and metastasis in $4 \mathrm{~T} 1$ breast cancer. Biomaterials. 2019;217:13. doi:10.1016/j.biomaterials.2019.119264

26. Vankayala R, Huang YK, Kalluru P, Chiang CS, Hwang KC. First demonstration of gold nanorods-mediated photodynamic therapeutic destruction of tumors via near infra-red light activation. Small. 2014;10(8):1612-1622. doi:10.1002/smll.201302719

27. Gai SL, Yang GX, Yang PP, et al. Recent advances in functional nanomaterials for light-triggered cancer therapy. Nano Today. 2018;19:146-187. doi:10.1016/j.nantod.2018.02.010

28. Costa DF, Mendes LP, Torchilin VP. The effect of low- and high-penetration light on localized cancer therapy. Adv Drug Deliv Rev. 2019;138:105-116. doi:10.1016/j.addr.2018.09.004

29. Ntziachristos V, Yodh AG, Schnall M, Chance B. Concurrent MRI and diffuse optical tomography of breast after indocyanine green enhancement. Proc Natl Acad Sci U S A. 2000;97(6):2767-2772. doi:10.1073/pnas.040570597

30. Chu KF, Dupuy DE. Thermal ablation of tumours: biological mechanisms and advances in therapy. Nat Rev Cancer. 2014;14 (3):199-208. doi:10.1038/nrc3672

31. Zhang CW, Zhao XZ, Guo SH, Lin TS, Guo HQ. Highly effective photothermal chemotherapy with $\mathrm{pH}$-responsive polymer-coated drug-loaded melanin-like nanoparticles. Int $J$ Nanomedicine. 2017;12:1827-1840. doi:10.2147/ijn.S130539

32. Zhang D, Wu M, Zeng YY, et al. Chlorin e6 conjugated poly(dopamine) nanospheres as PDT/PTT dual-modal therapeutic agents for enhanced cancer therapy. ACS Appl Mater Interfaces. 2015;7 (15):8176-8187. doi:10.1021/acsami.5b01027

33. Liu YL, Ai KL, Liu JH, Deng M, He YY, Lu LH. Dopamine-melanin colloidal nanospheres: an efficient near-infrared photothermal therapeutic agent for in vivo cancer therapy. Adv Mater. 2013;25 (9):1353-1359. doi:10.1002/adma.201204683

34. Park J, Brust TF, Lee HJ, Lee SC, Watts VJ, Yeo Y. Polydopamine-based simple and versatile surface modification of polymeric nano drug carriers. ACS Nano. 2014;8(4):3347-3356. doi:10.1021/nn405809c

35. Zhang XY, Wang SQ, Xu LX, et al. Biocompatible polydopamine fluorescent organic nanoparticles: facile preparation and cell imaging. Nanoscale. 2012;4(18):5581-5584. doi:10.1039/c2nr31281f

36. LaVoie MJ, Ostaszewski BL, Weihofen A, Schlossmacher MG, Selkoe DJ. Dopamine covalently modifies and functionally inactivates parkin. Nat Med. 2005;11(11):1214-1221. doi:10.1038/nm1314

37. Lee H, Rho J, Messersmith PB. Facile conjugation of biomolecules onto surfaces via mussel adhesive protein inspired coatings. $A d v$ Mater. 2009;21(4):431-443. doi:10.1002/adma.200801222

38. Li H, Jia Y, Peng HN, Li JB. Recent developments in dopamine-based materials for cancer diagnosis and therapy. $A d v$ Colloid Interface Sci. 2018;252:1-20. doi:10.1016/j.cis.2018.01.001

39. Roberts R, Hanna L, Borley A, Dolan G, Williams EM. Epirubicin chemotherapy in women with breast cancer: alternating arms for intravenous administration to reduce chemical phlebitis. Eur J Cancer Care. 2019;28(5):9. doi:10.1111/ecc.13114
40. Khasraw M, Bell R, Dang C. Epirubicin: is it like doxorubicin in breast cancer? A clinical review. Breast. 2012;21(2):142-149. doi:10.1016/j.breast.2011.12.012

41. Ryberg M, Nielsen D, Cortese G, Nielsen G, Skovsgaard T, Andersen PK. New insight into epirubicin cardiac toxicity: competing risks analysis of 1097 breast cancer patients. J Natl Cancer Inst. 2008;100(15):1058-1067. doi:10.1093/jnci/djn206

42. Gandhi R, Khatri N, Baradia D, Vhora I, Misra A. Surface-modified Epirubicin- $\mathrm{HCl}$ liposomes and its in vitro assessment in breast cancer cell-line: MCF-7. Drug Deliv. 2016;23(4):1152-1162. doi:10.3109/ 10717544.2014.999960

43. Pan Q, Zhang J, Li X, et al. Preparation and pharmacokinetics of bifunctional epirubicin-loaded micelles. Pharmazie. 2019;74 (10):577-582. doi:10.1691/ph.2019/9059

44. Zhang J, Chen YC, Li X, Liang XL, Luo XJ. The influence of different long-circulating materials on the pharmacokinetics of liposomal vincristine sulfate. Int J Nanomedicine. 2016;11:4187-4197. doi:10.2147/ijn.S109547

45. Zhang J, Miao L, Guo S, et al. Synergistic anti-tumor effects of combined gemcitabine and cisplatin nanoparticles in a stroma-rich bladder carcinoma model. J Control Release. 2014;182:90-96. doi:10.1016/j.jconrel.2014.03.016

46. Diehl KH, Hull R, Morton D, et al. A good practice guide to the administration of substances and removal of blood, including routes and volumes. J Appl Toxicol. 2001;21(1):15-23. doi:10.1002/jat.727

47. Gaber MH, Hong KL, Huang SK, Papahadjopoulos D. Thermosensitive sterically stabilized liposomes: formulation and in vitro studies on mechanism of doxorubicin release by bovine serum and human plasma. Pharm Res. 1995;12(10):1407-1416. doi:10.1023/a:1016206631006

48. Niu PH, Li FF, Liang XJ, et al. A porous polyaniline nanotube sorbent for solid-phase extraction of the fluorescent reaction product of reactive oxygen species in cells, and its determination by HPLC. Mikrochim Acta. 2018;185(10):468. doi:10.1007/s00604-018-3000-6

49. Ying WH. NAD ${ }^{+}$and NADH in cellular functions and cell death. Front Biosci. 2006;11(1):3129-3148. doi:10.2741/2038

50. Poinard B, Neo SZY, Yeo ELL, Heng HPS, Neoh KG, Kah JCY. Polydopamine nanoparticles enhance drug release for combined photodynamic and photothermal therapy. ACS Appl Mater Interfaces. 2018;10(25):21125-21136. doi:10.1021/ acsami.8b04799

51. Della Vecchia NF, Luchini A, Napolitano A, et al. Tris buffer modulates polydopamine growth, aggregation, and paramagnetic properties. Langmuir. 2014;30(32):9811-9818. doi:10.1021/ la501560z

52. Wust P, Hildebrandt B, Sreenivasa G, et al. Hyperthermia in combined treatment of cancer. Lancet Oncol. 2002;3(8):487-497. doi:10.1016/s1470-2045(02)00818-5

53. Field SB, Bleehen NM. Hyperthermia in the treatment of cancer. Cancer Treat Rev. 1979;6(2):63-94. doi:10.1016/S0305-7372(79) 80043-2

54. Wang Z, Duan Y, Duan YW. Application of polydopamine in tumor targeted drug delivery system and its drug release behavior. $J$ Control Release. 2018;290:56-74. doi:10.1016/j.jconrel.2018.10.009

55. Ding YP, Su SS, Zhang RR, et al. Precision combination therapy for triple negative breast cancer via biomimetic polydopamine polymer core-shell nanostructures. Biomaterials. 2017;113:243-252. doi:10.1016/j.biomaterials.2016.10.053

56. Wang MX, You CQ, Gao ZG, et al. A dual-targeting strategy for enhanced drug delivery and synergistic therapy based on thermosensitive nanoparticles. $J$ Biomater Sci Polym Ed. 2018;29 (11):1360-1374. doi:10.1080/09205063.2018.1460141

57. Morgenstern J, Baumann P, Brunner C, Hubbuch J. Effect of PEG molecular weight and PEGylation degree on the physical stability of PEGylated lysozyme. Int $J$ Pharm. 2017;519(1-2):408-417. doi:10.1016/j.ijpharm.2017.01.040 
58. Rafiee E, Eavani S. pH-responsive controlled release of epirubicin from Fe@Si-PW hybrid nanoparticles. Mater Sci Eng C Mater Biol Appl. 2014;39:340-343. doi:10.1016/j.msec.2014.03.020

59. Bhojoo U, Chen MH, Zou S. Temperature induced lipid membrane restructuring and changes in nanomechanics. Biochim Biophys Acta. 2018;1860(3):700-709. doi:10.1016/j.bbamem.2017.12.008

60. Jiang YY, Li JC, Zhen X, Xie C, Pu KY. Dual-peak absorbing semiconducting copolymer nanoparticles for first and second near-infrared window photothermal therapy: a comparative study. Adv Mater. 2018;30(14):7. doi:10.1002/adma.201705980

61. Zhang FR, Liu YH, Lei JN, et al. Metal-organic-framework-derived carbon nanostructures for site-specific dual-modality photothermal/ photodynamic thrombus therapy. Adv Sci. 2019;6(17):8. doi:10.1002/ advs.201901378

62. Liu WL, Liu T, Zou MZ, et al. Aggressive man-made red blood cells for hypoxia-resistant photodynamic therapy. Adv Mater. 2018;30 (35):10. doi:10.1002/adma.201802006

63. Wang SH, Shang L, Li LL, et al. Metal-organic-framework-derived mesoporous carbon nanospheres containing porphyrin-like metal centers for conformal phototherapy. Adv Mater. 2016;28(38):8379-8387. doi:10.1002/adma.201602197

64. Tian N, Sun WZ, Guo XS, et al. Mitochondria targeted and NADH triggered photodynamic activity of chloromethyl modified $\mathrm{Ru}(\mathrm{ii})$ complexes under hypoxic conditions. Chem Commun. 2019;55 (18):2676-2679. doi:10.1039/c8cc09186b
65. Green DR, Reed JC. Mitochondria and apoptosis. Science. 1998;281 (5381):1309-1312. doi:10.1126/science.281.5381.1309

66. Hennig R, Kuespert S, Haunberger A, Goepferich A, Fuchshofer R. Cyclic RGD peptides target human trabecular meshwork cells while ameliorating connective tissue growth factor-induced fibrosis. J Drug Target. 2016;24(10):952-959. doi:10.3109/1061186X.2016.1163709

67. Zhong YN, Meng FH, Deng C, Zhong ZY. Ligand-directed active tumor-targeting polymeric nanoparticles for cancer chemotherapy. Biomacromolecules. 2014;15(6):1955-1969. doi:10.1021/bm5003009

68. Otsuka H, Nagasaki Y, Kataoka K. PEGylated nanoparticles for biological and pharmaceutical applications. Adv Drug Deliv Rev. 2003;55(3):403-419. doi:10.1016/s0169-409x(02)00226-0

69. Zhang J, Shen LM, Li X, Song WT, Liu Y, Huang L. Nanoformulated codelivery of quercetin and alantolactone promotes an antitumor response through synergistic immunogenic cell death for microsatellite-stable colorectal cancer. ACS Nano. 2019;13 (11):12511-12524. doi:10.1021/acsnano.9b02875

70. Mi Y, Liu Y, Feng SS. Formulation of docetaxel by folic acidconjugated d- $\alpha$-tocopheryl polyethylene glycol succinate 2000 (Vitamin E TPGS2k) micelles for targeted and synergistic chemotherapy. Biomaterials. 2011;32(16):4058-4066. doi:10.1016/j. biomaterials.2011.02.022

71. Liu JP, Sun J, Zhang N, Jiang C. Biopharmaceutics and Pharmacokinetics (5th Edition). Beijing: People's Medical Publishing House; 2016:92.
International Journal of Nanomedicine

\section{Publish your work in this journal}

The International Journal of Nanomedicine is an international, peerreviewed journal focusing on the application of nanotechnology in diagnostics, therapeutics, and drug delivery systems throughout the biomedical field. This journal is indexed on PubMed Central, MedLine, CAS, SciSearch ${ }^{\circledR}$, Current Contents ${ }^{\circledR} /$ Clinical Medicine,

\section{Dovepress}

Journal Citation Reports/Science Edition, EMBase, Scopus and the Elsevier Bibliographic databases. The manuscript management system is completely online and includes a very quick and fair peer-review system, which is all easy to use. Visit http://www.dovepress.com/ testimonials.php to read real quotes from published authors. 